\title{
Cell autonomous TGF-beta signaling is essential for cell recruitment into degenerating tendons
}

2 Guak-Kim Tan ${ }^{1,2,{ }^{*}}$, Brian A. Pryce ${ }^{1}$, Anna Stabio ${ }^{1}$, Douglas R. Keene ${ }^{1}$, Sara F. Tufa ${ }^{1}$ and Ronen

3 Schweitzer ${ }^{1,2, *}$

$4{ }^{1}$ Research Division, Shriners Hospital for Children, Portland, OR 97239, USA.

$5 \quad{ }^{2}$ Department of Orthopaedics and Rehabilitation, School of Medicine, Oregon Health \& Science

6 University, Portland, OR 97239, USA.

7 * Authors for correspondence (tagu@ohsu.edu; schweitz@ohsu.edu)

\section{Abstract}

11 Understanding the role of cell recruitment in tendon disorders is critical for improvements in

12 regenerative therapy. We recently reported that targeted disruption of TGF $\beta$ type II receptor in 13 the tendon cell lineage $\left(T g f b r 2^{S c x C r e}\right)$ resulted in tenocyte dedifferentiation and tendon 14 degradation in post-natal stages. Here we extend the analysis and identify direct recruitment of 15 stem/progenitor cells into the degenerative mutant tendons. Cre-lineage tracing indicates that these cells are not derived from tendon ensheathing tissues or from a Scleraxis-lineage, and they

17 turned on tendon markers only upon entering the mutant tendons. Through immunohistochemistry and inducible gene deletion, we further find that the recruited cells

19 originated from a Sox9-expressing lineage and their recruitment was dependent on cell20 autonomous TGF $\beta$ signaling. These results thus differ from previous reports of cell recruitment 21 into injured tendons, and suggest a critical role for TGF $\beta$ signaling and cell recruitment in the 22 etiology and treatment of tendon degeneration. 


\section{$\underline{\text { Introduction }}$}

Response to tissue damage or pathology commonly involves the activation or recruitment of stem/progenitor cells that help to replenish the tissue and, in many cases, participate in the healing response (Rennert et al., 2012). While some stem/progenitor cells reside within the tissue, e.g. satellite cells in muscle (Yin et al., 2013), in other scenarios they are recruited from an external niche (Mathews et al., 2004; Jujo et al., 2010; Xynos et al., 2010). Direct detection and investigation of tissue specific stem/progenitor cells was revolutionized by the advent of Cre technology that facilitated the ability to label specific cell populations and monitor their involvement in healing and pathology (Kan et al., 2018; Harvey et al., 2019). The emerging theme is one where for each tissue there are various types of stem/progenitor cells that participate in such processes and likely reflect differential responses to different types of injury or pathology (Rinkevich et al., 2011; Marecic et al., 2015). Identifying the specific cells that participate in the healing response and the signals involved in their activation and recruitment is critical for progress in efforts to enhance and improve the clinical outcomes of therapy. In this study, we identify a new type of progenitor cell involved in the response to tendon pathology.

Tendons are type I collagen rich tissues that transmit the forces generated by muscle contraction to bone (Kannus, 2000). The considerable mechanical challenges to tendons result in a high frequency of injuries that range from acute damage, e.g. in tendon laceration, to chronic damage due to overuse and tissue degeneration as in tendinopathy (Sharma and Maffulli, 2005; Cook et al., 2016). The considerable burden of tendon injuries to individuals and society is compounded by the slow and frequently poor healing of these tissues that often results in impaired tissue integrity (Gerber et al., 2000; Boileau et al., 2005). A better understanding of biological processes underlying tendon healing may thus provide insight towards more effective therapies for tendon injuries.

Experimental investigation on tendon repair has mainly focused on acute injury using transected animal tendons (Forslund and Aspenberg, 2003; Ferry et al., 2007; Howell et al., 2017). Just like other tissues within the body, it has been suggested that cells involved in tendon healing can be from both intrinsic and extrinsic sources (Biro and Bihari-Varga, 1974; Harrison et al., 2003; Jones et al., 2003; Howell et al., 2017). In the latter scenario, recent studies have shown that 
some of the recruited cells express stem/progenitor markers (Dyment et al., 2014; Runesson et al., 2015; Wang et al., 2017; Harvey et al., 2019). Moreover, several groups have reported that stem/progenitor cells can be isolated from the surrounding peritenon (i.e. epitenon and paratenon) and tendon sheath (Mienaltowski et al., 2013; Wang et al., 2017), and suggest that these tissues may be a source of the recruited cells. Indeed, by taking advantage of Cre/loxP reporter system for cell lineage tracing, Dyment et al reported that alpha-smooth muscle actin $(\alpha-S M A)-$ positive paratenon cells are the major contributor to the healing response following patellar tendon injury (Dyment et al., 2014). Other lineage tracing experiments have indicated the potential involvement of TPPP3 and osteocalcin-expressing cells during tendon repair (Wang et al., 2017; Harvey et al., 2019). Moreover, injured mouse Achilles tendon was found to be infiltrated by stem/progenitor cells that exhibited different regional distribution and temporal expression (Runesson et al., 2015), implying the existence of multiple recruited cell populations. Despite this recent progress in understanding of the healing response in tendons, basic biology of recruited cells including identity, signals responsible for recruitment, and their role at the injured site, remains largely unknown.

We recently reported that disruption of TGF $\beta$ signaling in the tendon cell lineage by targeting TGF $\beta$ type II receptor gene Tgfbr2 using the ScxCre driver (Tgfbr2 ${ }^{S c x C r e}$ ) resulted in tenocyte dedifferentiation in early postnatal stages. Tendon cells in Tgfbr $2^{S c x c r e}$ mutants appeared normal during embryogenesis, but in early postnatal stages lost all differentiation markers including Scx, tenomodulin and collagen I (Tan et al., 2020). Extending the analysis of these mutant tendons, we now find that mutant tendons also began to show progressive degenerative changes, a feature frequently observed in tendinopathy (Kannus and Jozsa, 1991; Longo et al., 2018). Moreover, we find that cells with stem/progenitor features were recruited into the deteriorating mutant tendons. The recruited cells originated from a Sox9-expressing lineage, and we further demonstrate that TGF $\beta$ signaling was essential for their recruitment. Additionally, it appears that these cells are different from those reported in other studies of cell recruitment into tendons, tendon. 


\section{$\underline{\text { Results }}$}

\section{ScxGFP-expressing cells in Tgfbr $2^{\text {ScxCre }}$ mutant tendons are newly recruited}

87 Targeting of the TGF $\beta$ type II receptor gene Tgfbr2 with ScxCre resulted in a dramatic tendon phenotype (Tan et al., 2020). In early postnatal stages, a few lateral tendons disintegrated and snapped, while in the majority of the tendons, the tenocytes lost the tendon cell fate and dedifferentiated (Figure 1A) (Tan et al., 2020). Macroscopically, the mutant tendons appeared grey and thin, in contrast to normal tendons in which the tight organization of the collagen fibers results in a brilliant white color with firm texture (Figure 1B). The underlying changes were examined by transmission electron microscopy (TEM) and histological analyses that revealed disorganization of collagen matrix, severe disruption of the epitenon structure and paratenon thickening (Figure 1C-G). These tendons may therefore share some similarities with tendons in various pathological conditions (Longo et al., 2008; Dyment et al., 2013).

While the majority of cells in the degenerating tendons of $T g f b r 2^{\text {ScxCre }}$ mutants lost expression of ScXGFP and other tendon differentiation markers by P7 (Figure 2A, black arrowhead) (Tan et al., 2020), we observed the appearance of cells that contrary to the surrounding cells expressed ScxGFP (Figure 2A, white arrowhead). The ScxGFP-positive cells in mutant tendons also expressed other prototypic tendon markers tenomodulin and Col1a1 (Figure 2E,F, white arrowheads). Despite the induction of tendon markers, these cells differ morphologically from normal tenocytes at this stage. P7 wild-type tenocytes display a stellar-like morphology and a rectangular shape in transverse and longitudinal sections, respectively (Figure 2B,D, black arrowheads). In contrast, the ScxGFP-positive cells appeared large and rounded in both views (Figure 2A,C, white arrowheads). Factors that contribute to the aberrant morphology of these cells were not identified to date.

Surprisingly, some of these ScxGFP-positive cells exhibited weak or no expression of the Cre reporter Ai14 Rosa26-tdTomato (RosaT) (Figure 2G). Conversely, all tendon cells in P7 Tgfbr $2^{f /+} ; S c x C r e$ heterozygous pups were marked by robust RosaT expression (Figure $2 \mathrm{H}$ ). ScxGFP and ScxCre are transgenic mice that utilize the Scleraxis enhancer to drive expression of eGFP and 112 Cre respectively (Pryce et al., 2007; Blitz et al., 2013). In mice that carry both constructs, e.g. the Tgfbr $2^{\text {ScxCre }}$ mutant, it is likely that ScxGFP signal will be detected first upon Scx activation, since 
114

115

116

117

118

119

120

121

122

123

124

125

126

127

128

129

130

131

132

detection of RosaT requires an intermediate step of protein synthesis. First Cre activity has to reach threshold levels to induce reporter recombination followed by a second step in which the reporter signal is accumulated to achieve detectable levels. Based on this logic, we hypothesized that ScxGFP-positive but RosaT-negative cells in mutant tendons (called hereafter ScxGFP+;RosaT-) are cells from non Scx-expressing cell lineage in which the Scx enhancer was recently activated, i.e. they were newly recruited into the mutant tendons. Indeed, when ScxGFP+;RosaT - cells were isolated by fluorescence activated cell sorting (FACS) and cultured, they subsequently also showed expression of the RosaT reporter (Supplementary Figure 1).

Direct detection of cell recruitment in the process of tendon healing, as demonstrated by this observation, is exciting because it may open new directions for analysis of the healing response. We therefore wanted to reinforce this result with an approach that will identify newly recruited cells by a positive signal rather than the absence of expression of a reporter. To achieve this goal we repeated the experiment utilizing the $m T m G$ dual fluorescent Cre reporter in which the ubiquitously expressed membrane-tomato $(m T)$ is replaced by membrane-GFP $(m G)$ upon Cremediated recombination (Figure 3A) (Muzumdar et al., 2007). The advantage of this reporter system over RosaT is that it allows a simultaneous visualization and determination of both the recombined and non-recombined states. As expected, in P7 tendons, $m$ TmG cells were labelled red $(m T)$ in the absence of Cre activity (Figure 3B), while all tendon cells were recombined and appeared positive for $m G$ in ScxCre;mTmG pups (Figure 3C). On the other hand, in tendons of the Tgfbr ${ }^{\text {ScxCre }}$ mutant some of the ScxGFP-positive cells had a recombined Cre reporter (Figure 3D, white arrowhead), whereas others retained the $m T$ signal indicating they did not recombine the reporter or at least have not yet lost the $m T$ signal (Figure 3D, yellow arrowhead).

To further evaluate Cre activity in the ScxGFP-positive cells we attempted to detect expression of the TGF $\beta$ type II receptor protein and indeed found expression of the receptor in some of these cells (Figure 3E, black arrowhead). Taken together, these observations reflect a recent induction of Scx expression in the ScxGFP-positive cells, suggesting these cells are newly recruited into the mutant tendons. 


\section{Temporal dynamics of cell recruitment into mutant tendons}

There are only a handful of reports of cell recruitment into tendons (Dyment et al., 2013; Wang et al., 2017; Harvey et al., 2019; Kaji et al., 2020), and almost nothing is known about the origin of such cells or the mechanisms of their recruitment. A robust method for detecting such cells as ScxGFP+;RosaT- cells in the tendons of $T g f b r 2^{\text {ScxCre }}$ mutants therefore provided a unique opportunity to learn more about this process. In tendons of wild-type pups with the same marker combination, nearly all cells were positive for both ScxGFP and RosaT while ScxGFP+;RosaT-cells were very rare (Figure 4A), suggesting this marker combination indeed provides a robust approach for identifying newly recruited cells.

Utilizing this approach, we found that the newly recruited cells (i.e. ScxGFP+;RosaT-) could be detected already at P1 in the tendons of mutant pups (Figure 4B, blue circle). The levels of these cells within mutant tendons peaked between P1 to P3 and remained detectable throughout the observation period (results not shown). Significantly, in these early postnatal stages most mutant tendons were intact and did not show structural indications of damage (Tan et al., 2020).

Moreover, since tissue repair involves early recruitment of immune and inflammatory cells to the damaged site (Millar et al., 2010; Kragsnaes et al., 2014), we wanted to determine if the recruited cells were associated with an immune response and thus examined for the presence of relevant markers. In both P1 and P7 mutant tendons, we found only a small number of cells expressing the activated macrophage marker F4/80 (Figure 5A). Notably, there was no noticeable difference in their numbers compared to normal tendons (Figure 5B). Moreover, mutant tendons stained negatively for the inflammatory marker TNF- $\alpha$ (Figure 5C), as also observed in normal tendons (Figure 5D). Cell recruitment into the tendons of $T g f b r 2^{S c x C r e}$ mutants thus initiated prior to any sign of a structural destruction or immune response, suggesting a specific molecular signal and not general tissue damage may be the driver of cell recruitment in this case. 


\section{The recruited cells do not originate from peritenon or the tendon sheath}

A handful of recent studies identified cell recruitment into tendons mostly in the context of injury (Dyment et al., 2013; Tan et al., 2013; Runesson et al., 2015; Wang et al., 2017) and possibly also following physiological loading (Mendias et al., 2012). While the origin of such cells remains unclear, it was suggested in a few studies that they may arise from the peritenon (i.e. paratenon and epitenon) or tendon sheath (Dyment et al., 2013; Wang et al., 2017; Harvey et al., 2019). To assess if the recruited cells in Tgfbr $2^{S c x C r e}$ mutant tendons were derived from peritenon or tendon sheath, we again took advantage of the RosaT Cre reporter system. Not much is known about gene expression in these tendon ensheathing tissues that clearly do not overlap with gene expression in tenocytes (Harvey et al., 2019; Tan et al., 2020). Interestingly, while peritenon and tendon sheath cells do not express the tendon reporter ScxGFP they are consistently positive for RosaT Cre reporter in mice carrying ScxCre; RosaT alleles (Figure 4C). This combination of markers likely represents transient expression of Scx in early progenitor cells of the peritenon and tendon sheath that was sufficient for activation of the Cre reporter. Since the newly recruited cells are from non Scx-expressing lineage and do not express the Cre reporter RosaT as noted earlier (Figure 4B), the cells recruited into the mutant tendons were not derived from peritenon and tendon sheath.

Notably, the newly recruited ScxGFP+;RosaT-cells could also be detected in the peritenon and tendon sheath of $T g f b r 2^{S c x C r e}$ mutant pups (Figure 4D, blue circles). Since the absence of Cre reporter expression indicates that these are not original peritenon or tendon sheath cells, we postulate that these are either the recruited cells entering the mutant tendons by passing through peritenon and tendon sheath, or cells also being recruited into these tendon ensheathing tissues in mutant pups.

Additionally, previous studies have demonstrated the invasion of cells expressing $\alpha$-SMA, also a pericyte marker, into injured tendons with a likely endothelial-perivascular origin (Dyment et al., 2013; Howell et al., 2017). To determine if this may also be the origin of the recruited cells in the tendons of $T g f b r 2^{S c x C r e}$ mutants we examined expression of endothelial and pericyteassociated markers (Cathery et al., 2018), but could not detect expression of CD31, CD146 or $\alpha$ SMA in these cells (data not shown). Taken together these results suggest that the cells getting 
recruited into the tendons of $T g f b r 2^{S c x c r e}$ mutants are different from the cells so far reported to be implicated in tendon injury, and possibly represent a repair response to the pathological (degenerating) changes in the Tgfbr2 $2^{\text {ScxCre }}$ mutant tendons.

\section{The recruited cells have clonogenic features and express stem/progenitor markers}

Direct detection and the ability to isolate the newly recruited cells into the mutant tendons presented a unique opportunity to characterize the cellular features and possibly origin of the recruited cells. We hypothesized that for participation in tendon healing the recruited cells likely have features of stem/progenitor cells and tested for such features. Wild-type tendons contain $2-4 \%$ cells with colony forming potential, also known as tendon-derived stem/progenitor cells (TSPC) (Bi et al., 2007; Mienaltowski et al., 2013) (Figure 6B). To test the colony forming capacity of the recruited cells we dissociated cells from the tendons of P7 mutant pups and isolated the recruited cells by FACS based on the unique marker combination of these cells (SCXGFP+;RosaT-). The cells were seeded at one cell per well in 96-well plates and colony forming potential was determined after 9 to 14 days of culture (Figure 6A). We indeed found that $2.9 \pm 0.7 \%$ of the recruited cells had colony forming potential ( $n=4$ independent experiments in duplicate) (Figure $6 \mathrm{~B})$, but the size of clones formed by the recruited cells varied and in general was smaller than the clones of wild-type TSPC (Figure 6C). The difference presumably reflects the dynamic change of cellular state in the recruited cells, in which some cells were newly recruited and still possessed progenitor stemness features, while others have already advanced in assuming the tendon cell fate and thus lost their proliferative capacity to give rise to large colonies.

Recognizing the progenitor state of the recruited cells, we next tested these cells in P7 mutant tendons for expression of typical markers identified in cultured TSPC and other established progenitor markers (Bi et al., 2007; Mienaltowski et al., 2013; Lui, 2015). We first found that the recruited cells expressed the stem/progenitor marker nucleostemin, which is not expressed by normal tendon cells (Figure 7A,C) (Zhang and Wang, 2010). Surprisingly, we also detected expression of Sox9 protein in the recruited cells (Figure 7D). Sox9 is most commonly recognized as an early cartilage marker but it is also expressed in various populations of stem/progenitor 
227 cells (Poche et al., 2008; Scott et al., 2010; Furuyama et al., 2011). It was previously demonstrated 228 that some tendon progenitors express Sox9, and some Sox9CreERT2 activity can be detected in 229 tenocytes even in postnatal stages (Soeda et al., 2010; Blitz et al., 2013; Huang et al., 2019). 230 However, expression of Sox9 protein is undetectable or negligible by immunohistochemistry in 231 normal tenocytes (Figure 7B) and is therefore unique to these recruited cells. Notably, the 232 number of Sox9- and nucleostemin-positive cells was high at P1, coinciding with the earliest 233 detectable recruitment in mutant tendons (Figure 4B) and the prevalence of these cells gradually 234 declined in later stages. Taken together, our results suggest that cells with stem/progenitor 235 features were recruited into mutant tendons. Additionally, the recruited cells expressing the 236 ScxGFP reporter were not detected away from the tendons but only within or adjacent to mutant 237 tendons (Supplementary Figure 2), suggesting that irrespective of their origin the cells turned on 238 tendon gene expression only upon entering the tissue.

\section{Cell autonomous TGF $\beta$ signaling is essential for cell recruitment into mutant tendons}

241 TGF $\beta$ signaling has been implicated in cell motility and recruitment in other systems (Franitza et 242 al., 2002; Tang et al., 2009) and recently also in tendons (Kaji et al. 2020). Since we found that the recruited cells still expressed the TGF $\beta$ type II receptor, we next wanted to ask if deletion of Tgfbr2 in these cells will change their capacity for recruitment. We previously found that the tendon phenotype in $T g f b r 2^{S c x C r e}$ mutants is dependent on the specific spatio-temporal features of ScxCre activity (Tan et al., 2020). To target the Tgfbr2 receptor before the cells are recruited, we therefore decided to add the ubiquitous inducible Cre deletor (RosaCreERT2) (Hameyer et al., 2007) to the $T g f b r 2^{S c x C r e}$ allele combination. Since the tendon phenotype manifests in $T g f b r 2^{S c x C r e}$ mutants in postnatal stages, we can use RosaCreERT2 to induce ubiquitous loss of the receptor at that stage and examine the effect on cell recruitment into mutant tendons.

251 Pups of the mutant allele combination, Tgfbr $2^{f /-} ;$ ScxCre; RosaCreERT2 (called hereafter 252 ScxCre;RosaCreERT Double Cre mutant), were given tamoxifen at the earliest time-point of 253 detectable recruitment at P1 and P2, and harvested at P7. Interestingly, we found nearly 60\% 254 reduction in the number of recruited cells in the ScxCre;RosaCreERT Double Cre mutant pups 
compared to $T g f b r 2^{S c x C r e}$ mutants (Figure $\left.8 \mathrm{~A}-\mathrm{C}\right)(p<0.01 ; \mathrm{n}=3)$. The dramatic reduction in cell recruitment suggests that TGF $\beta$ signaling indeed plays a role in cell recruitment. However, the partial reduction of recruitment in these experiments may also imply the existence of alternative molecular mechanisms or may simply reflect partial Cre activation. Tamoxifen application in neonates has severe deleterious effects. It was therefore not possible to increase the dosage or number of days in which tamoxifen was administered. To test if Cre activity was partial in the ScxCre;RosaCreERT Double Cre mutants, we stained mutant forelimb sections with anti-TGF $\beta$ type II receptor antibody. Intriguingly, cells that were still recruited into the mutant tendons in the Double Cre experiment were positive for the receptor (Figure 8D, arrowheads), suggesting a complete dependence of cell recruitment on TGF $\beta$ signaling. Moreover, the fact that Tgfbr 2 expressing cells could still be recruited in this scenario demonstrates that the loss of TGF $\beta$ signaling did not have a general effect on the capacity of cells to be recruited or on the recruiting signal, but rather TGF $\beta$ signaling acts cell-autonomously and was required for the ability of individual cells to be recruited in this scenario.

The identity and anatomical origin of the recruited cells is of great importance for future efforts to manipulate and enhance the healing processes. The experimental paradigm used above provided us with a unique tool to test hypotheses regarding the origin of the recruited cells in this experimental model, since we can use various other inducible Cre lines with a more restricted target population to target the receptor and test the effects on cell recruitment. We demonstrated above that most of the newly recruited cells expressed the Sox9 protein (Figure 7D). It was important, however, to determine if Sox9 expression was induced only during the recruitment process or if it was expressed in the cells prior to their recruitment and therefore may be used as a marker to identify the origin of these cells. We therefore employed the same Double Cre strategy to target Tgfbr2 but in this time specifically in Sox9-expressing cells using a Sox9CreERT2 driver in combination with the Tgfbr2 $2^{S c x C r e}$ mutant (Tgfbr2 ${ }^{f /-}$;ScxCre;Sox9CreERT2, called hereafter ScxCre;Sox9CreERT Double Cre mutant). The results showed about $48 \%$ decrease in recruited cell numbers $(p<0.01 ; n=3$ ) (Figure $8 C$ ), suggesting that most if not all of the recruited cells indeed expressed Sox9 prior to their activation. 


\section{$\underline{\text { Discussion }}$}

285

286

287

288

289

290

291

292

293

294

295

296

297

298

299

300

301

302

303

304

305

306

307

308

309

310

311

312

The present studies extend our previous observations where targeted disruption of TGF $\beta$ signaling in tendon cells (i.e. Scx-expressing cell lineage) led to loss of their cell fate (Tan et al., 2020), and provide three major observations. First, we find in post-natal stages a progressive tissue degeneration in the mutant tendons, a condition that has been often associated with tendinopathy and spontaneous tendon rupture (Kannus and Jozsa, 1991; Jarvinen et al., 1997; Longo et al., 2008). Secondly, we identify direct recruitment of stem/progenitor cells into the deteriorating tendons. Furthermore, findings from the Cre-lineage tracing indicate that these cells are not derived from surrounding peritenon or tendon sheath, implying the existence of multiple sources for stem/progenitor cell recruitment in tendons. Thirdly, we find that most if not all of the recruited cells were from Sox9-expressing lineage, and TGF $\beta$ signaling is essential for their recruitment into the degenerating tendons. This scenario thus opens an opportunity to directly examine the origin of recruited stem/progenitor cells and the mechanisms of their activation in degenerative tendon pathologies.

In mutant tendons, cell recruitment was at a pick already at P1, in the absence of observable structural damage or immune response in these tendons, suggesting that the process of tenocyte dedifferentiation in mutant tendons is also accompanied by the secretion of a specific recruitment signal. We therefore suggest the following model for cell recruitment in this scenario (Figure 9): (1) Tenocyte dedifferentiation in neonatal mutants results also in secretion of a stem/progenitor cell recruitment and/or activation signal(s). (2,3) Activation and/or recruitment of the stem/progenitor cells is dependent on activation of TGF $\beta$ signaling in these cells in a cell autonomous manner. TGF $\beta$ ligands may therefore be the recruitment signals in this case. It may however also be possible that a different signal is employed for cell recruitment and TGF $\beta$ signaling plays an essential role in the activation or motility of the cells towards the degenerating tendons. (4) Expression of ScxGFP in the recruited cells is observed only in or near the target tendons, suggesting the induction of the tendon cell fate in these cells is not an integral part of the activation or recruitment process, but rather that an additional local signal or interaction with the tendon cells or environment lead to induction of the tendon cell fate in the recruited cells while they integrate into the mutant tendon. 
Tendon damage occurs very frequently, but the tissue tends to heal poorly (Gerber et al., 2000; Boileau et al., 2005). Therefore, there is great interest in the use of stem/progenitor cells to improve tendon repair and therapy (Nourissat et al., 2010; Hernigou et al., 2014; Oh et al., 2014). Previous investigations have demonstrated that acute tendon injury involves recruitment 317 of new cells expressing stem/progenitor markers including $\alpha$-SMA, Oct-3/4, TPPP3 and osteocalcin (Dyment et al., 2013; Runesson et al., 2015; Howell et al., 2017; Wang et al., 2017; Harvey et al., 2019). However, their relevance to degenerative tendons, a feature that precedes and underlies tendinopathy and tendon rupture, remains unclear. To our knowledge this is the

321 first demonstration of stem/progenitor cell recruitment in the context of a degenerative tendon 322 condition, thus opening new avenues for direct identification and analysis of stem/progenitor cells in vivo in the context of tendon pathology.

We show herein that the cells recruited into mutant tendons are clonogenic, and the majority of newly recruited cells are stained positive for nucleostemin and Sox9. Nucleostemin is a GTPbinding protein expressed predominantly in the nucleoli of stem/progenitor cells (Beekman et al., 2006; Nomura et al., 2009). In recent years, nucleostemin has been shown to be expressed by culture-expanded stem/progenitor cells from tendons (Zhang and Wang, 2010). Notably, infiltration of nucleostemin-positive progenitor cells into ruptured rat Achilles tendons has been reported in an earlier study (Runesson et al., 2015). Likewise, Sox9 is a transcription factor that is associated with various adult progenitor cell populations (Formeister et al., 2009; Furuyama et al., 2011) . Lineage tracing on Sox9CreERT2 mice suggest that Sox9-expressing cells also serve as progenitors during tendon development (Soeda et al., 2010; Huang et al., 2019). Importantly, numerous studies have reported the involvement of Sox9-positive stem/progenitor cells in tissue repair (Furuyama et al., 2011; He et al., 2017). Our results therefore suggest that Sox9 is also expressed in the niche of the stem/progenitor cells identified in this study and expression of Sox 9 may therefore serve as an initial indicator for possible location and origins of the cells.

Studies have suggested several possible sources of recruited cells into damaged tendons including peritenon (i.e. paratenon and epitenon) and tendon sheath (Dyment et al., 2013; Wang 340 et al., 2017; Harvey et al., 2019). Apparent changes of epitenon cellular activity, e.g. increased 341 proliferation, has been observed in many cases of tendon injuries (Khan et al., 1996). Moreover, 
recent studies show appearance and subsequent migration of ScxGFP-positive cells from paratenon into tendons following injury (Dyment et al., 2013; Sakabe et al., 2018). A similar phenomenon was observed in this study and prompted us to ask if the recruited cells were derived from these tissues. The results from ScxCre-lineage tracing indicate that these cells are derived neither from peritenon or tendon sheath regions. Moreover, the eventual structural destruction of epitenon in our mutant pups may also limit the availability of cells recruited from this region. These results therefore imply the existence of multiple sources of recruited stem/progenitor cells for tendons. Different sources for cell recruitment may reflect specialization of specific cells for different tendon conditions or the concurrent activation of multiple cell populations that may have complementing activities in the healing process. Interestingly, a previous study has shown biphasic infiltration of two different stem/progenitor cell populations into ruptured rat Achilles tendons (Runesson et al., 2015).

To better understand the identity and origin of the recruited cells, further investigation focused on Sox9 because immunostaining demonstrated robust Sox9 expression in these cells during the early phase of recruitment. Results from Double Cre experiment showed that inducible deletion of TGF $\beta$ signaling in Sox9-expressing cells significantly reduced the number of recruited cells in mutant pups. The finding not only corroborates our earlier notion that the recruited cells expressed Sox9, but further reveals that at least a subpopulation of the recruited cells is from Sox9-expressing cell lineage. Sox9CreERT-lineage tracing shows the presence of Sox9expressing cells in perichondrium and bone marrow in neonates, suggesting the possibility of these tissues as sources of the recruited stem/progenitor cells. Notably, stem/progenitor cells have been identified in the perichondrium and bone marrow, and these cells are involved in tissue repair (Pineault et al., 2019). The possible involvement in these perichondrial cells in tendon healing will be addressed in future studies.

Studies of stem/progenitor cells and their roles in normal development and pathology were revolutionized with the advent of Cre technology and the ability to label distinct cell populations with a combination of a tissue specific Cre driver and a Cre reporter. These studies are typically prospective, a hypothesis regarding the role of a specific cell population is tested by labeling these cells using a tissue specific Cre (Soeda et al., 2010; Wang et al., 2017; Harvey et al., 2019). 
371 In this study we developed a complementary retrospective approach to identify cell recruitment

372 into tendons. ScxGFP is a robust tendon reporter that results in strong GFP expression shortly

373 after activation of the Scx enhancer (Pryce et al., 2007). Activation of the RosaT reporter in a

374 ScxCre;RosaT combination requires two rounds of protein synthesis; First accumulation of

375 sufficient Cre protein and then after recombination and activation of the reporter, accumulation

376 of the reporter protein (Madisen et al., 2010). It is therefore likely that upon induction of the Scx

377 enhancer in a cell with no tenogenic history the ScxGFP signal will be detected first and the RosaT

378 signal will follow with some delay. Notably, this approach will not identify cells from the tendon sheathing tissues or intrinsic tendon cells, since these cells are from Scx-expressing cell lineage and thus will have an activated RosaT reporter. We suggest however that retrospective screening for ScxGFP+;RosaT-cells in tendons of ScxCre;RosaT;ScxGFP-carrying mice can be used as a general approach for identification of non Scx-expressing cell recruitment into tendons following injury or pathology.

Understanding key players that mediate cell recruitment into pathologic tendons is critical to design tendon reparative strategies. At present almost nothing is known about this process both in vitro and in vivo. Here we show that TGF $\beta$ signaling is essential for the cell recruitment into the degenerating mutant tendon, in which disruption of TGF $\beta$ type II receptor in these cells significantly reduced their number in the mutant tendons. TGF $\beta$ signaling is known to be involved in the recruitment of various cell types including stem/progenitor cells in pathologic conditions. For instance, blockage of TGF $\beta$ signaling with the receptor inhibitor abolished the mobilization and recruitment of mesenchymal stem cells to the injured arteries in mice (Wan et al., 2012). With regard to tendons, activation of TGF $\beta$ signaling pathway has been reported during embryonic tendon cell development (Havis et al., 2014). Moreover, a number of studies have demonstrated increased TGF $\beta$ ligand and receptor expression by tendon (Fenwick et al., 2001; Dahlgren et al., 2005; Favata et al., 2006) or its adjacent tissues (Khan et al., 1996) in pathological conditions. Interestingly, TGF $\beta$ seems to play an important role in mediating tendon repair (Chen et al., 2004) although the exact mechanism remains unclear. More recently, Kaji et al (Kaji et al., 2020) reported that TGF $\beta$ signaling is required in neonatal tenocytes for their recruitment to the site of tendon transection, and may play a role in promoting neonatal tendon regeneration. 
Notably, this study identified a role for TGF $\beta$ signaling for recruitment of the resident tenocytes into the wound site and suggested a possible additional cell population involved in this process. In the present study we provide evidence for a distinctly different role for TGF $\beta$ signaling in tendon pathology, i.e. recruitment of a separate population of stem/progenitor cells from a distant niche into the degenerating tendon. Interestingly, these results highlight repeated involvement of TGF $\beta$ signaling in distinct cellular events in tendon biology.

Our results also indicate a cell autonomous requirement of TGF $\beta$ signaling for the recruitment. In the Double Cre experiment with RosaCreERT2, the Tgfbr2 receptor was eliminated from all cells. The failure of cell recruitment in this scenario could therefore be the result of a role for TGF $\beta$ signaling in the recruiting tendon, the environment surrounding the tendon or in the recruited cells themselves. However, as shown in Figure 8D, individual cells that did not lose receptor expression were recruited in this scenario, suggesting that the disruption was not in the tendon or tendon environment, since that would affect all cell recruitment, but rather a direct effect on the stem/progenitor cells that lost Tgfbr2 expression. Future studies will focus on the mechanism of action at the cellular level of TGF $\beta$ signaling by transcriptome analysis of the recruited cells.

Lastly, TGF $\beta$ signaling is often associated with collagen matrix production (Klein et al., 2002; Leask and Abraham, 2004). However, apparent collagen disorganization was not observed in Tgfbr2 ${ }^{\text {ScxCre }}$ mutant tendons at the onset of the cellular phenotype (Tan et al., 2020). Instead, collagen disorganization and epitenon deterioration were noted only in pups older than oneweek. The degenerative changes might thus imply a secondary consequence of the cellular changes in these mutants and/or of their movement difficulties. Regardless of the underlying causes, degenerative change has been implicated as a feature of tendinopathy (Kannus and Jozsa, 1991; Jarvinen et al., 1997; Longo et al., 2018). Much of what we learn about tendon healing comes from studies on acute tendon injury (Dyment et al., 2013; Wang et al., 2017; Kaji et al., 2020), and their significance to healing of tendinopathic tendons remains questionable. Moreover, it is difficult to obtain early tendinopathic human tissues because the conditions are often initially asymptomatic. The degenerative phenotype in $T g f b r 2^{S c x C r e}$ mutant thus warrants 
428 further investigation and may provide a useful animal model for analysis of early degenerative 429 changes in tendons.

\section{Materials and Methods}

432 Mice

433 Floxed TGF $\beta$ type II receptor (Tgfbr $2^{f / f}$ ) mice (Chytil et al., 2002) were crossed with mice carrying 434 the tendon deletor Scleraxis-Cre recombinase (ScxCre) (Blitz et al., 2013) to disrupt TGF $\beta$ 435 signalling in tenocytes. The generation of Rosa26-CreERT2 (RosaTCreERT2) (Hameyer et al., 2007) 436 and Sox9CreERT2 (Soeda et al., 2010) mice have been described previously. All mice in this study 437 also carried a transgenic tendon reporter ScxGFP (Pryce et al., 2007), and Cre reporters Ai14 438 Rosa26-tdTomato (RosaT) (Madisen et al., 2010) or mTmG (Muzumdar et al., 2007) for lineage 439 tracing of Scx-expressing cells. All animal procedures were approved by the by the Animal Care 440 and Use Committee at Oregon Health \& Science University (OHSU). For embryo harvest, timed 441 mating was set up in the afternoon, and identification of a mucosal plug on the next morning was 442 considered 0.5 days of gestation (E0.5). Embryonic day 14.5 to postnatal day 13 (E14.5-P13) limb 443 tendons were used for analysis. The mice genotype was determined by PCR analysis of DNA 444 extracted from tail snip using a lysis reagent (Viagen Biotech, Cat 102-T) and proteinase $\mathrm{K}$ 445 digestion $\left(55^{\circ} \mathrm{C}\right.$, overnight).

\section{Transmission electron microscopy (TEM)}

448 Mouse forelimbs were skinned, fixed in 1.5\% glutaraldehyde/1.5\% formaldehyde and decalcified 449 in 0.2M EDTA. TEM was then performed as previously described (Tan et al., 2020). The acquired 450 images were stitched using ImageJ software (https://imagej.nih.gov/ij/) (Preibisch et al., 2009).

\section{In situ hybridization and immunohistochemistry staining}

453 Dissected forelimbs were fixed, decalcified and incubated with a 5-30\% sucrose/PBS gradient. 454 The tissues were then embedded in OCT (Tissue-Tek, Inc) and sectioned at 10-12 $\mu \mathrm{m}$ using a 
Microm HM550 cryostat (Thermo Scientific, Waltham, MA). In situ hybridization and immunohistochemistry staining were performed as previously described (Tan et al., 2020). For all qualitative studies, sections from two to four pups were examined to ensure reproducibility of results.

\section{Double Cre experiment to investigate the role of TGF $\beta$ signaling in cell recruitment}

CreERT2 recombinase was incorporated into the background of $\mathrm{Tgfbr}^{\mathrm{f} /-}$;ScxCre mutant to enable induction of either ubiquitous (RosaCreERT2) or restricted (Sox9CreERT2) Tgfbr2 deletion upon tamoxifen administration. The pups were administered orally with tamoxifen $(50 \mathrm{mg} / \mathrm{ml}$ in autoclaved corn oil; $15 \mu$ per pup) at P1 and P2 and harvested at P7, a stage at which only recruited cells are positive for ScxGFP in the mutant tendon.

\section{Quantification of recruited cells in transverse sections of the communis tendon}

For the analysis of the effect of TGF $\beta$ signaling ablation on cell recruitment, the number of recruited cells were counted in extensor digitorium communis tendons at the zeugopod level of both P7 Tgfbr2 ${ }^{\text {ScxCre }}$ and Double Cre (ScxCre;RosaCreERT and ScxCre;Sox9CreERT) mutant pups. Forelimb transverse sections were stained DAPI to highlight the nuclei for identifying the individual cell. Recruited cells were identified by positive ScxGFP signal at this stage and their number was counted in 6-15 sections per pup from 3-5 pups.

\section{Statistical analysis}

Student's $t$-tests were performed to determine the statistical significance of differences between groups $(n \geq 3)$. Unless stated otherwise, all graphs are presented as mean \pm standard deviation (SD). A value of $p<0.05$ is regarded as statistically significant. 


\section{Acknowledgement}

483

484

485

486

487

488

489

490

491

492

493

494

495

496

497

498

499

500

501

502

503

504

505

506

507

508

509

510

511

512

513

514

The authors thank Dr Elazar Zelzer (Department of Molecular Genetics, Weizmann Institute of Science, Israel) for critical reading of the manuscript. We are grateful to staff from FACS Core, OHSU particularly Dr Miranda Gilchrist for their excellent technical assistance. This work was funded by NIH (R01AR055973) and Shriners Hospital for Children (SHC 5410-POR-14). G.K.T was supported by Research Fellowship from Shriners Hospital for Children.

\section{Competing interests}

The authors declare no competing interests.

\section{References}

Beekman, C., Nichane, M., De Clercq, S., Maetens, M., Floss, T., Wurst, W., Bellefroid, E. and Marine, J. C. (2006) 'Evolutionarily conserved role of nucleostemin: controlling proliferation of stem/progenitor cells during early vertebrate development', Molecular and cellular biology 26(24): 9291-301.

Bi, Y., Ehirchiou, D., Kilts, T. M., Inkson, C. A., Embree, M. C., Sonoyama, W., Li, L., Leet, A. I., Seo, B. M., Zhang, L. et al. (2007) 'Identification of tendon stem/progenitor cells and the role of the extracellular matrix in their niche', Nature medicine 13(10): 1219-27.

Biro, T. and Bihari-Varga, M. (1974) '[Thermoanalytic studies in connective tissue research. II. Changes in the structure of cartilage and tendon tissues under pathologic conditions]', Magyar traumatologia, orthopaedia es helyreallito sebeszet 17(4): 282-6.

Blitz, E., Sharir, A., Akiyama, H. and Zelzer, E. (2013) 'Tendon-bone attachment unit is formed modularly by a distinct pool of Scx- and Sox9-positive progenitors', Development 140(13): 268090.

Boileau, P., Brassart, N., Watkinson, D. J., Carles, M., Hatzidakis, A. M. and Krishnan, S. G. (2005) 'Arthroscopic repair of full-thickness tears of the supraspinatus: does the tendon really heal?', The Journal of bone and joint surgery. American volume 87(6): 1229-40.

Cathery, W., Faulkner, A., Maselli, D. and Madeddu, P. (2018) 'Concise Review: The Regenerative Journey of Pericytes Toward Clinical Translation', Stem cells 36(9): 1295-1310.

Chen, Y. J., Wang, C. J., Yang, K. D., Kuo, Y. R., Huang, H. C., Huang, Y. T., Sun, Y. C. and Wang, F. S. (2004) 'Extracorporeal shock waves promote healing of collagenase-induced Achilles tendinitis and increase TGF-beta1 and IGF-I expression', Journal of orthopaedic research : official publication of the Orthopaedic Research Society 22(4): 854-61. 
Chytil, A., Magnuson, M. A., Wright, C. V. and Moses, H. L. (2002) 'Conditional inactivation of the TGF-beta type II receptor using Cre:Lox', Genesis 32(2): 73-5.

Cook, J. L., Rio, E., Purdam, C. R. and Docking, S. I. (2016) 'Revisiting the continuum model of tendon pathology: what is its merit in clinical practice and research?', Br J Sports Med 50(19): 1187-91.

Dahlgren, L. A., Mohammed, H. O. and Nixon, A. J. (2005) 'Temporal expression of growth factors and matrix molecules in healing tendon lesions', Journal of orthopaedic research : official publication of the Orthopaedic Research Society 23(1): 84-92.

Dyment, N. A., Hagiwara, Y., Matthews, B. G., Li, Y., Kalajzic, I. and Rowe, D. W. (2014) 'Lineage tracing of resident tendon progenitor cells during growth and natural healing', PLoS One 9(4): e96113.

Dyment, N. A., Liu, C. F., Kazemi, N., Aschbacher-Smith, L. E., Kenter, K., Breidenbach, A. P., Shearn, J. T., Wylie, C., Rowe, D. W. and Butler, D. L. (2013) 'The paratenon contributes to scleraxisexpressing cells during patellar tendon healing', PLoS One 8(3): e59944.

Favata, M., Beredjiklian, P. K., Zgonis, M. H., Beason, D. P., Crombleholme, T. M., Jawad, A. F. and Soslowsky, L. J. (2006) 'Regenerative properties of fetal sheep tendon are not adversely affected by transplantation into an adult environment', Journal of orthopaedic research : official publication of the Orthopaedic Research Society 24(11): 2124-32.

Fenwick, S. A., Curry, V., Harrall, R. L., Hazleman, B. L., Hackney, R. and Riley, G. P. (2001) 'Expression of transforming growth factor-beta isoforms and their receptors in chronic tendinosis', Journal of anatomy 199(Pt 3): 231-40.

Ferry, S. T., Dahners, L. E., Afshari, H. M. and Weinhold, P. S. (2007) 'The effects of common antiinflammatory drugs on the healing rat patellar tendon', The American journal of sports medicine 35(8): 1326-33.

Formeister, E. J., Sionas, A. L., Lorance, D. K., Barkley, C. L., Lee, G. H. and Magness, S. T. (2009) 'Distinct SOX9 levels differentially mark stem/progenitor populations and enteroendocrine cells of the small intestine epithelium', American journal of physiology. Gastrointestinal and liver physiology 296(5): G1108-18.

Forslund, C. and Aspenberg, P. (2003) 'Improved healing of transected rabbit Achilles tendon after a single injection of cartilage-derived morphogenetic protein-2', The American journal of sports medicine 31(4): 555-9.

Franitza, S., Kollet, O., Brill, A., Vaday, G. G., Petit, I., Lapidot, T., Alon, R. and Lider, O. (2002) 'TGFbeta1 enhances SDF-1alpha-induced chemotaxis and homing of naive $T$ cells by up-regulating CXCR4 expression and downstream cytoskeletal effector molecules', European journal of immunology 32(1): 193-202.

Furuyama, K., Kawaguchi, Y., Akiyama, H., Horiguchi, M., Kodama, S., Kuhara, T., Hosokawa, S., Elbahrawy, A., Soeda, T., Koizumi, M. et al. (2011) 'Continuous cell supply from a Sox9-expressing progenitor zone in adult liver, exocrine pancreas and intestine', Nature genetics 43(1): 34-41. 
Gerber, C., Fuchs, B. and Hodler, J. (2000) 'The results of repair of massive tears of the rotator cuff', The Journal of bone and joint surgery. American volume 82(4): 505-15.

Hameyer, D., Loonstra, A., Eshkind, L., Schmitt, S., Antunes, C., Groen, A., Bindels, E., Jonkers, J., Krimpenfort, P., Meuwissen, R. et al. (2007) 'Toxicity of ligand-dependent Cre recombinases and generation of a conditional Cre deleter mouse allowing mosaic recombination in peripheral tissues', Physiological genomics 31(1): 32-41.

Harrison, R. K., Mudera, V., Grobbelaar, A. O., Jones, M. E. and McGrouther, D. A. (2003) 'Synovial sheath cell migratory response to flexor tendon injury: an experimental study in rats', The Journal of hand surgery 28(6): 987-93.

Harvey, T., Flamenco, S. and Fan, C. M. (2019) 'A Tppp3(+)Pdgfra(+) tendon stem cell population contributes to regeneration and reveals a shared role for PDGF signalling in regeneration and fibrosis', Nature cell biology 21(12): 1490-1503.

Havis, E., Bonnin, M. A., Olivera-Martinez, I., Nazaret, N., Ruggiu, M., Weibel, J., Durand, C., Guerquin, M. J., Bonod-Bidaud, C., Ruggiero, F. et al. (2014) 'Transcriptomic analysis of mouse limb tendon cells during development', Development 141(19): 3683-96. positive periosteal cells in fracture repair of the adult mammalian long bone', Bone 103: 12-19. Rouard, H. (2014) 'Biologic augmentation of rotator cuff repair with mesenchymal stem cells during arthroscopy improves healing and prevents further tears: a case-controlled study', Int 573 Orthop 38(9): 1811-8.

574 Howell, K., Chien, C., Bell, R., Laudier, D., Tufa, S. F., Keene, D. R., Andarawis-Puri, N. and Huang, 575 A. H. (2017) 'Novel Model of Tendon Regeneration Reveals Distinct Cell Mechanisms Underlying 576 Regenerative and Fibrotic Tendon Healing', Scientific reports 7: 45238.

577 Huang, A. H., Watson, S. S., Wang, L., Baker, B. M., Akiyama, H., Brigande, J. V. and Schweitzer, R. 578 (2019) 'Requirement for scleraxis in the recruitment of mesenchymal progenitors during 579 embryonic tendon elongation', Development 146(20).

580 Jarvinen, M., Jozsa, L., Kannus, P., Jarvinen, T. L., Kvist, M. and Leadbetter, W. (1997) 581 'Histopathological findings in chronic tendon disorders', Scandinavian journal of medicine \& 582 science in sports 7(2): 86-95.

583 Jones, M. E., Mudera, V., Brown, R. A., Cambrey, A. D., Grobbelaar, A. O. and McGrouther, D. A. 584 (2003) 'The early surface cell response to flexor tendon injury', The Journal of hand surgery 28(2): 585 221-30.

586 Jujo, K., Hamada, H., Iwakura, A., Thorne, T., Sekiguchi, H., Clarke, T., Ito, A., Misener, S., Tanaka, 587 T., Klyachko, E. et al. (2010) 'CXCR4 blockade augments bone marrow progenitor cell recruitment 588 to the neovasculature and reduces mortality after myocardial infarction', Proceedings of the 589

590 Kaji, D. A., Howell, K. L., Balic, Z., Hubmacher, D. and Huang, A. H. (2020) 'Tgfbeta signaling is 591 required for tenocyte recruitment and functional neonatal tendon regeneration', Elife 9. 
Kan, C., Chen, L., Hu, Y., Ding, N., Li, Y., McGuire, T. L., Lu, H., Kessler, J. A. and Kan, L. (2018) 'Gli1labeled adult mesenchymal stem/progenitor cells and hedgehog signaling contribute to endochondral heterotopic ossification', Bone 109: 71-79.

Kannus, P. (2000) 'Structure of the tendon connective tissue', Scandinavian journal of medicine \& science in sports 10(6): 312-20.

Kannus, P. and Jozsa, L. (1991) 'Histopathological changes preceding spontaneous rupture of a tendon. A controlled study of 891 patients', The Journal of bone and joint surgery. American volume 73(10): 1507-25.

Khan, U., Edwards, J. C. and McGrouther, D. A. (1996) 'Patterns of cellular activation after tendon injury', J Hand Surg Br 21(6): 813-20.

Klein, M. B., Yalamanchi, N., Pham, H., Longaker, M. T. and Chang, J. (2002) 'Flexor tendon healing in vitro: effects of TGF-beta on tendon cell collagen production', The Journal of hand surgery 27(4): 615-20.

Kragsnaes, M. S., Fredberg, U., Stribolt, K., Kjaer, S. G., Bendix, K. and Ellingsen, T. (2014) 'Stereological quantification of immune-competent cells in baseline biopsy specimens from achilles tendons: results from patients with chronic tendinopathy followed for more than 4 years', The American journal of sports medicine 42(10): 2435-45.

Leask, A. and Abraham, D. J. (2004) 'TGF-beta signaling and the fibrotic response', FASEB journal : official publication of the Federation of American Societies for Experimental Biology 18(7): 81627.

Longo, U. G., Franceschi, F., Ruzzini, L., Rabitti, C., Morini, S., Maffulli, N. and Denaro, V. (2008) 'Histopathology of the supraspinatus tendon in rotator cuff tears', The American journal of sports medicine 36(3): 533-8.

Longo, U. G., Ronga, M. and Maffulli, N. (2018) 'Achilles Tendinopathy', Sports Med Arthrosc Rev 26(1): 16-30.

Lui, P. P. (2015) 'Markers for the identification of tendon-derived stem cells in vitro and tendon stem cells in situ - update and future development', Stem cell research \& therapy 6: 106.

Madisen, L., Zwingman, T. A., Sunkin, S. M., Oh, S. W., Zariwala, H. A., Gu, H., Ng, L. L., Palmiter, R. D., Hawrylycz, M. J., Jones, A. R. et al. (2010) 'A robust and high-throughput Cre reporting and characterization system for the whole mouse brain', Nature neuroscience 13(1): 133-40.

Marecic, O., Tevlin, R., McArdle, A., Seo, E. Y., Wearda, T., Duldulao, C., Walmsley, G. G., Nguyen, A., Weissman, I. L., Chan, C. K. et al. (2015) 'Identification and characterization of an injuryinduced skeletal progenitor', Proceedings of the National Academy of Sciences of the United States of America 112(32): 9920-5.

Mathews, V., Hanson, P. T., Ford, E., Fujita, J., Polonsky, K. S. and Graubert, T. A. (2004) 'Recruitment of bone marrow-derived endothelial cells to sites of pancreatic beta-cell injury', Diabetes 53(1): 91-8. 
Mendias, C. L., Gumucio, J. P., Bakhurin, K. I., Lynch, E. B. and Brooks, S. V. (2012) 'Physiological loading of tendons induces scleraxis expression in epitenon fibroblasts', J Orthop Res 30(4): 60612.

Mienaltowski, M. J., Adams, S. M. and Birk, D. E. (2013) 'Regional differences in stem cell/progenitor cell populations from the mouse achilles tendon', Tissue engineering. Part A 19(12): 199-210.

Millar, N. L., Hueber, A. J., Reilly, J. H., Xu, Y., Fazzi, U. G., Murrell, G. A. and Mclnnes, I. B. (2010) 'Inflammation is present in early human tendinopathy', The American journal of sports medicine 38(10): 2085-91.

Muzumdar, M. D., Tasic, B., Miyamichi, K., Li, L. and Luo, L. (2007) 'A global double-fluorescent Cre reporter mouse', Genesis 45(9): 593-605.

Nomura, J., Maruyama, M., Katano, M., Kato, H., Zhang, J., Masui, S., Mizuno, Y., Okazaki, Y., Nishimoto, M. and Okuda, A. (2009) 'Differential requirement for nucleostemin in embryonic stem cell and neural stem cell viability', Stem cells 27(5): 1066-76.

Nourissat, G., Diop, A., Maurel, N., Salvat, C., Dumont, S., Pigenet, A., Gosset, M., Houard, X. and Berenbaum, F. (2010) 'Mesenchymal stem cell therapy regenerates the native bone-tendon junction after surgical repair in a degenerative rat model', PLoS One 5(8): e12248.

Oh, J. H., Chung, S. W., Kim, S. H., Chung, J. Y. and Kim, J. Y. (2014) '2013 Neer Award: Effect of the adipose-derived stem cell for the improvement of fatty degeneration and rotator cuff healing in rabbit model', Journal of shoulder and elbow surgery 23(4): 445-55.

Pineault, K. M., Song, J. Y., Kozloff, K. M., Lucas, D. and Wellik, D. M. (2019) 'Hox11 expressing regional skeletal stem cells are progenitors for osteoblasts, chondrocytes and adipocytes throughout life', Nature communications 10(1): 3168.

Poche, R. A., Furuta, Y., Chaboissier, M. C., Schedl, A. and Behringer, R. R. (2008) 'Sox9 is expressed in mouse multipotent retinal progenitor cells and functions in Muller glial cell development', The Journal of comparative neurology 510(3): 237-50.

Preibisch, S., Saalfeld, S. and Tomancak, P. (2009) 'Globally optimal stitching of tiled 3D microscopic image acquisitions', Bioinformatics 25(11): 1463-5.

Pryce, B. A., Brent, A. E., Murchison, N. D., Tabin, C. J. and Schweitzer, R. (2007) 'Generation of transgenic tendon reporters, ScxGFP and ScxAP, using regulatory elements of the scleraxis gene', Developmental dynamics : an official publication of the American Association of Anatomists 236(6): 1677-82.

Rennert, R. C., Sorkin, M., Garg, R. K. and Gurtner, G. C. (2012) 'Stem cell recruitment after injury: lessons for regenerative medicine', Regenerative medicine 7(6): 833-50.

Rinkevich, Y., Lindau, P., Ueno, H., Longaker, M. T. and Weissman, I. L. (2011) 'Germ-layer and lineage-restricted stem/progenitors regenerate the mouse digit tip', Nature 476(7361): 409-13. 
Runesson, E., Ackermann, P., Karlsson, J. and Eriksson, B. I. (2015) 'Nucleostemin- and Oct 3/4positive stem/progenitor cells exhibit disparate anatomical and temporal expression during rat Achilles tendon healing', BMC Musculoskelet Disord 16: 212.

Sakabe, T., Sakai, K., Maeda, T., Sunaga, A., Furuta, N., Schweitzer, R., Sasaki, T. and Sakai, T. (2018) 'Transcription factor scleraxis vitally contributes to progenitor lineage direction in wound healing of adult tendon in mice', The Journal of biological chemistry 293(16): 5766-5780.

Scott, C. E., Wynn, S. L., Sesay, A., Cruz, C., Cheung, M., Gomez Gaviro, M. V., Booth, S., Gao, B., Cheah, K. S., Lovell-Badge, R. et al. (2010) 'SOX9 induces and maintains neural stem cells', Nature neuroscience 13(10): 1181-9.

Sharma, P. and Maffulli, N. (2005) 'Tendon injury and tendinopathy: healing and repair', The Journal of bone and joint surgery. American volume 87(1): 187-202.

Soeda, T., Deng, J. M., de Crombrugghe, B., Behringer, R. R., Nakamura, T. and Akiyama, H. (2010) 'Sox9-expressing precursors are the cellular origin of the cruciate ligament of the knee joint and the limb tendons', Genesis 48(11): 635-44.

Tan, G. K., Pryce, B. A., Stabio, A., Brigande, J. V., Wang, C., Xia, Z., Tufa, S. F., Keene, D. R. and Schweitzer, R. (2020) 'Tgfbeta signaling is critical for maintenance of the tendon cell fate', Elife 9.

Tan, Q., Lui, P. P. and Lee, Y. W. (2013) 'In vivo identity of tendon stem cells and the roles of stem cells in tendon healing', Stem Cells Dev 22(23): 3128-40.

Tang, Y., Wu, X., Lei, W., Pang, L., Wan, C., Shi, Z., Zhao, L., Nagy, T. R., Peng, X., Hu, J. et al. (2009) 'TGF-beta1-induced migration of bone mesenchymal stem cells couples bone resorption with formation', Nature medicine 15(7): 757-65.

Wan, M., Li, C., Zhen, G., Jiao, K., He, W., Jia, X., Wang, W., Shi, C., Xing, Q., Chen, Y. F. et al. (2012) 'Injury-activated transforming growth factor beta controls mobilization of mesenchymal stem cells for tissue remodeling', Stem cells 30(11): 2498-511.

Wang, Y., Zhang, X., Huang, H., Xia, Y., Yao, Y., Mak, A. F., Yung, P. S., Chan, K. M., Wang, L., Zhang, C. et al. (2017) 'Osteocalcin expressing cells from tendon sheaths in mice contribute to tendon repair by activating Hedgehog signaling', Elife 6.

Xynos, A., Corbella, P., Belmonte, N., Zini, R., Manfredini, R. and Ferrari, G. (2010) 'Bone marrowderived hematopoietic cells undergo myogenic differentiation following a Pax-7 independent pathway', Stem cells 28(5): 965-73.

Yin, H., Price, F. and Rudnicki, M. A. (2013) 'Satellite cells and the muscle stem cell niche', Physiological reviews 93(1): 23-67.

Zhang, J. and Wang, J. H. (2010) 'Characterization of differential properties of rabbit tendon stem cells and tenocytes', BMC musculoskeletal disorders 11: 10. 
bioRxiv preprint doi: https://doi.org/10.1101/2020.11.11.378505; this version posted November 11,2020 . The copyright holder for this preprint

(which was not certified by peer review) is the author/funder, who has granted bioRxiv a license to display the preprint in perpetuity. It is made available under aCC-BY-ND 4.0 International license.

\section{A $\mathbf{P 7}$ Het}

706

707

708

709

710

711

712

713

714

715

716
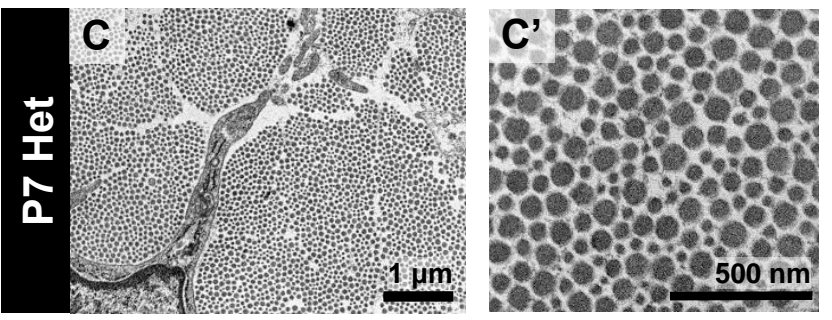

\section{B P7 Het}
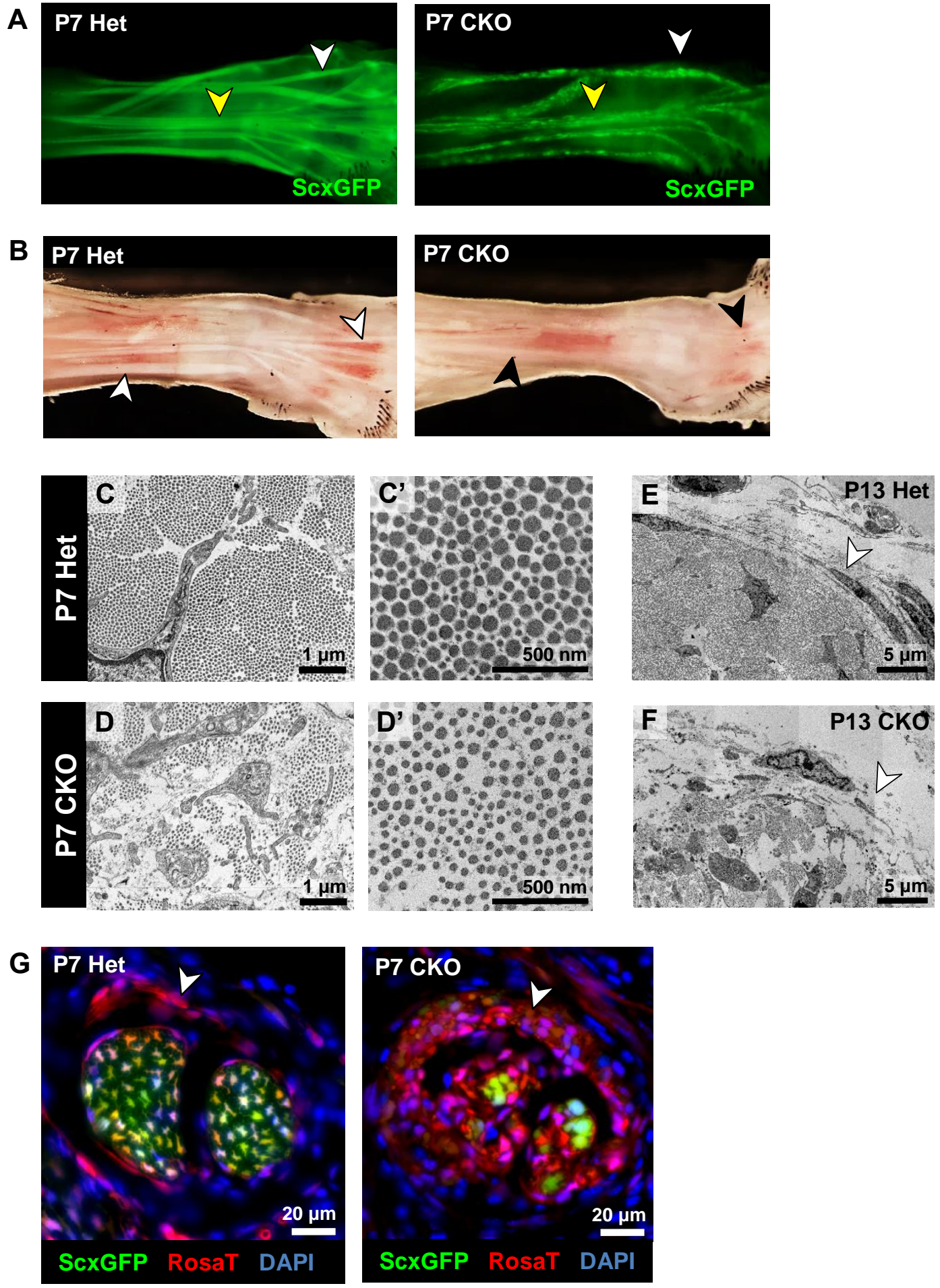

724

725 
727 Figure 1. Disruption of tendon structure in $\operatorname{Tgfbr}^{\text {ScxCre }}$ mutant. (A) Comparison of tendon

728 reporter ScxGFP signal in forelimbs from P7 heterozygous control ( $\mathrm{Ffbr}^{\mathrm{f} /+}$;ScxCre) and mutants

729 pups revealed that in mutants a few lateral tendons were missing (white arrowhead) and in the

730 other tendons there was a substantial loss of the ScXGFP signal (yellow arrowhead). (B) Brightfield

731 imaging of skinned forelimbs from P7 heterozygous control and mutant pups. While normal

732 tendons display a brilliant white color reflecting the tight organization of the collagen fibers

733 (white arrowheads), mutant tendons had a pale grey appearance (black arrowheads), likely

734 reflecting disruptions to the collagen matrix. (C-F) TEM analysis of heterozygous control and

735 mutant tendons. (C,D) In P7 normal tendons the collagen matrix was highly organized while in

736 mutant tendons the collagen fibrils were smaller with significant gaps between fibril bundles. C'

737 and $D^{\prime}$ are high-magnification images of collagen fibrils in C and D, respectively. (E-F) By P13, in

738 some regions of mutant tendons the epitenon was disrupted and discontinuous (white

739 arrowheads). (G) Triple labeling of transverse sections of heterozygous control and mutant

740 tendons using DAPI nuclear counterstain, ScxGFP and Cre reporter Ai14 Rosa26-tdTomato

741 (RosaT). RosaT labeling of the paratenon highlights significant thickening of the tissue observed

742 in some mutant tendons (white arrowheads). Mutant: CKO, Heterozygous: Het. 

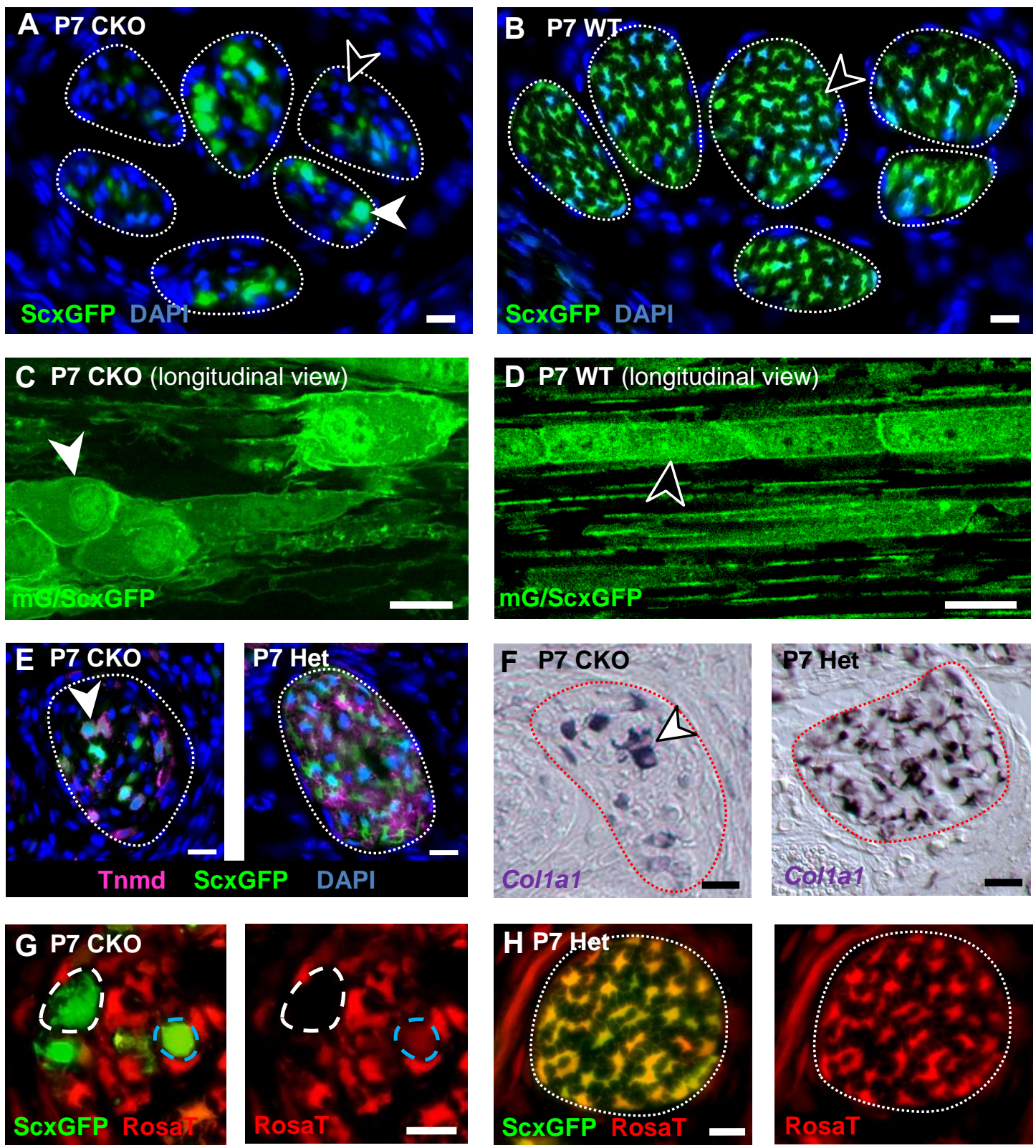

Figure 2. Morphology and marker expression of the ScxGFP-positive cells in Tgfbr2 $^{\text {ScxCre }}$ mutant

777 tendons. Transverse sections $(A, B, E-H)$ and longitudinal sections $(C, D)$ of tendons from forelimbs

778 of P7 wild-type and mutant pups. In all panels, ScxGFP identifies cells with tendon gene 779 expression and nuclear DAPI staining reflects general cellular distribution. $(A, B)$ In wild-type pups 780 all cells of the extensor digitorium communis (EDC) tendons expressed the ScxGFP reporter. 781 Conversely, the majority of cells in mutant tendons lost ScxGFP expression (black arrowhead), 782 but a small number of ScxGFP-expressing cells were also found in these tendons (white 783 arrowhead). Interestingly, while the wild-type tendon cells had a star-like morphology in 
transverse section, the ScxGFP-positive mutant tendon cells were significantly larger and had a round morphology. (C,D) Longitudinal sections from EDC tendons of P7 wild-type and mutant pups carrying the ScxGFP and ScxCre;mTmG reporters. While ScxGFP labels the cell body of tenocytes, ScxCre;mTmG results in membrane GFP signal to further accentuate the cell morphology (Muzumdar et al., 2007). Wild-type tenocytes were organized in prototypic cell rows and had a rectangular shape in longitudinal view (black arrowhead). However, the ScxGFPpositive cells in mutant tendons were rounded and the row organization was disrupted (white arrowhead). (E,F) The ScxGFP-positive cells in mutant tendons also expressed tendon markers tenomodulin and Col1a1. (E) Immunofluorescence for tenomodulin (Tnmd) in P7 forelimb tendons. Note that in heterozygous control tendons all tenocytes expressed Tnmd but in mutant tendons most cells lost Tnmd expression and only the ScxGFP-positive cells expressed Tnmd. (F) In situ hybridization for Col1a1 on tendons from P7 mutant and heterozygous pups. While all tendon cells expressed Col1a1 in heterozygous control, only a handful of large and rounded cells were positive in mutant tendons (white arrowhead). (G,H) ScxGFP and ScxCre;RosaT;ScxGFP expression in tendons from mutant and heterozygous control pups. (G) Some of the ScxGFPpositive cells exhibited weak or no expression of the Cre reporter RosaT (blue and white circles respectively), suggesting these cells may be newly recruited with a recent induction of $S c x .(H)$ Lower magnification image of representative P7 control tendons showing all tendon cells were marked by robust RosaT expression at this stage. Dashed lines demarcate tendons. Scale bar, 10 $\mu \mathrm{m}$. Mutant: CKO, Wild-type: WT, Heterozygous: Het.

(1)

7

.

09

10

11


A

817

818

819

820

821

822

823

824

825

826

827

828

829

830

831

832

833

834

835

836

837

838

839

840

841

842

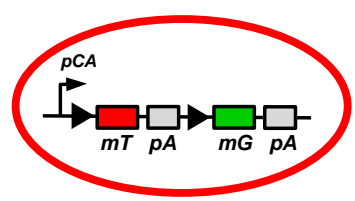

Non-recombined

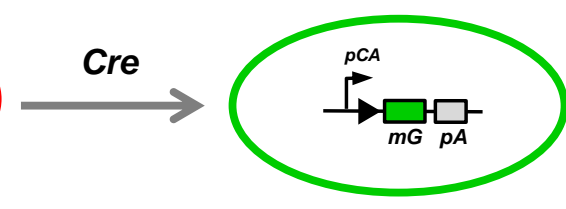

Recombined
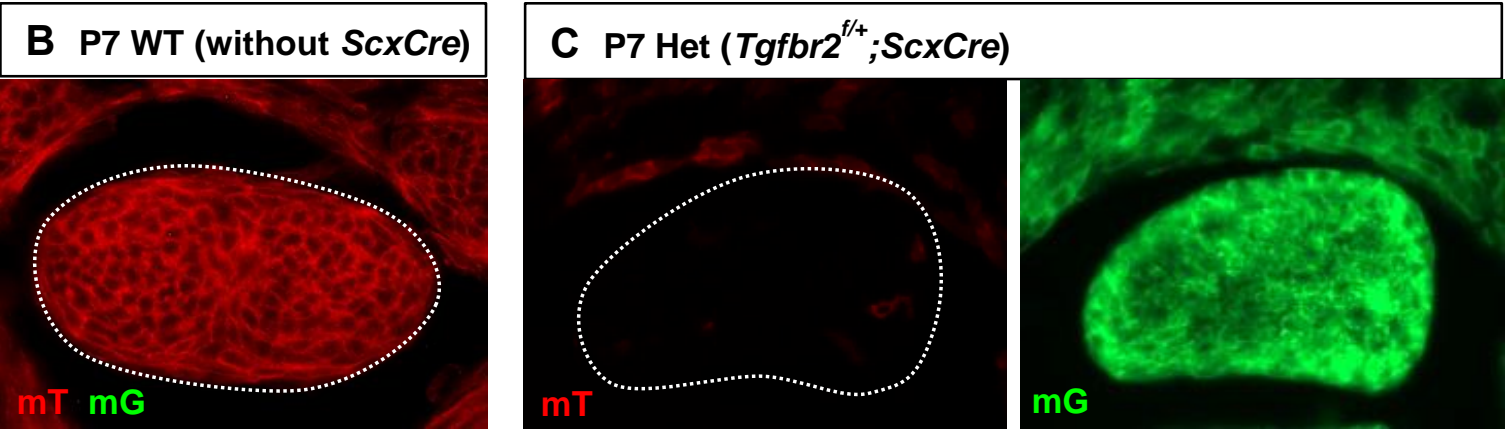

\section{P7 CKO}
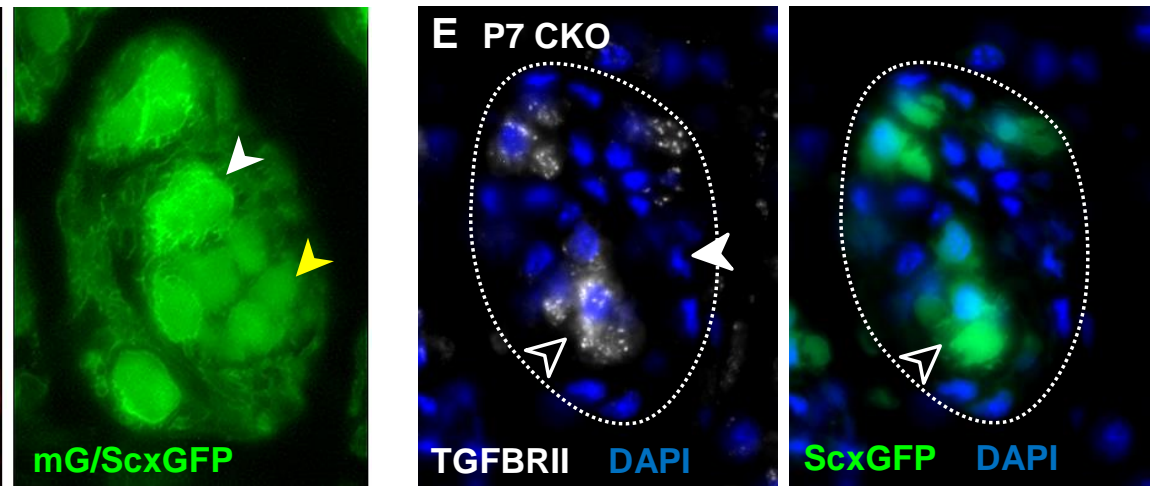

Figure 3. ScxGFP-expressing cells in $T g f b r 2^{S c x c r e}$ mutant tendons are newly recruited. (A) Schematic illustration of the mTmG dual-fluorescent Cre reporter (Muzumdar et al., 2007). Ubiquitously expressed membrane-tdTomato $(m T)$ is replaced by membrane-GFP $(m G)$ upon Cre-mediated recombination, allowing simultaneous detection of the recombined and the nonrecombined states. (B-E) Transverse sections of $\mathrm{P} 7$ forelimb tendons. $(\mathrm{B}, \mathrm{C}) m T m G$ expression in wild-type tendons. In the absence of Cre activity, red $(m T)$ fluorescence signal is detected in all cells including tendon cells. (C) In Tgfbr2 ${ }^{f /+} ; S c x C r e ; m T m G$ heterozygous pups, tendon cells were recombined and switched the fluorescence from red to green (mG). (D) In $\mathrm{Tgfbr}^{\mathrm{Scx} C \mathrm{Cre}}$ mutant tendons, the $m T m G$ Cre reporter was recombined in some of the ScxGFP-positive cells (white arrowheads) whereas the Cre reporter was not recombined in other ScxGFP-positive cells (yellow arrowhead). (E) Immunofluorescence staining with antibodies to the TGF $\beta$ type II receptor (TGFBRII) in mutant tendons. The resident tendon cells have completely lost expression of the receptor as expected at this stage (white arrowhead), but some of the ScxGFP-positive cells still 
bioRxiv preprint doi: https://doi.org/10.1101/2020.11.11.378505; this version posted November 11,2020 . The copyright holder for this preprint (which was not certified by peer review) is the author/funder, who has granted bioRxiv a license to display the preprint in perpetuity. It is made available under aCC-BY-ND 4.0 International license.

843 expressed the TGFBRII, further reinforcing the absence of Cre activity in these cells. Tendons are

844 demarcated by dashed lines. Mutant: CKO, Heterozygous: Het. Images not to scale.

845

846

847

848

849

850

851

852

853

854

855

856

857

858

859

860

861

862

863

864

865

866

867

868 


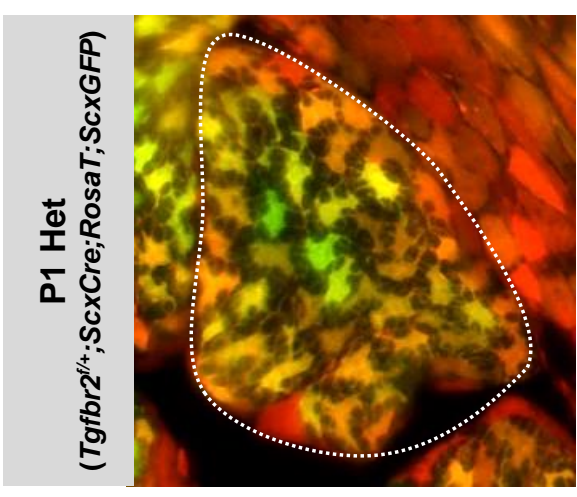
ScxGFP RosaT

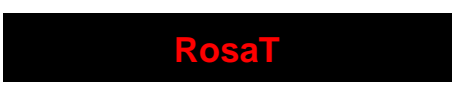

A

1

2

(2)

B
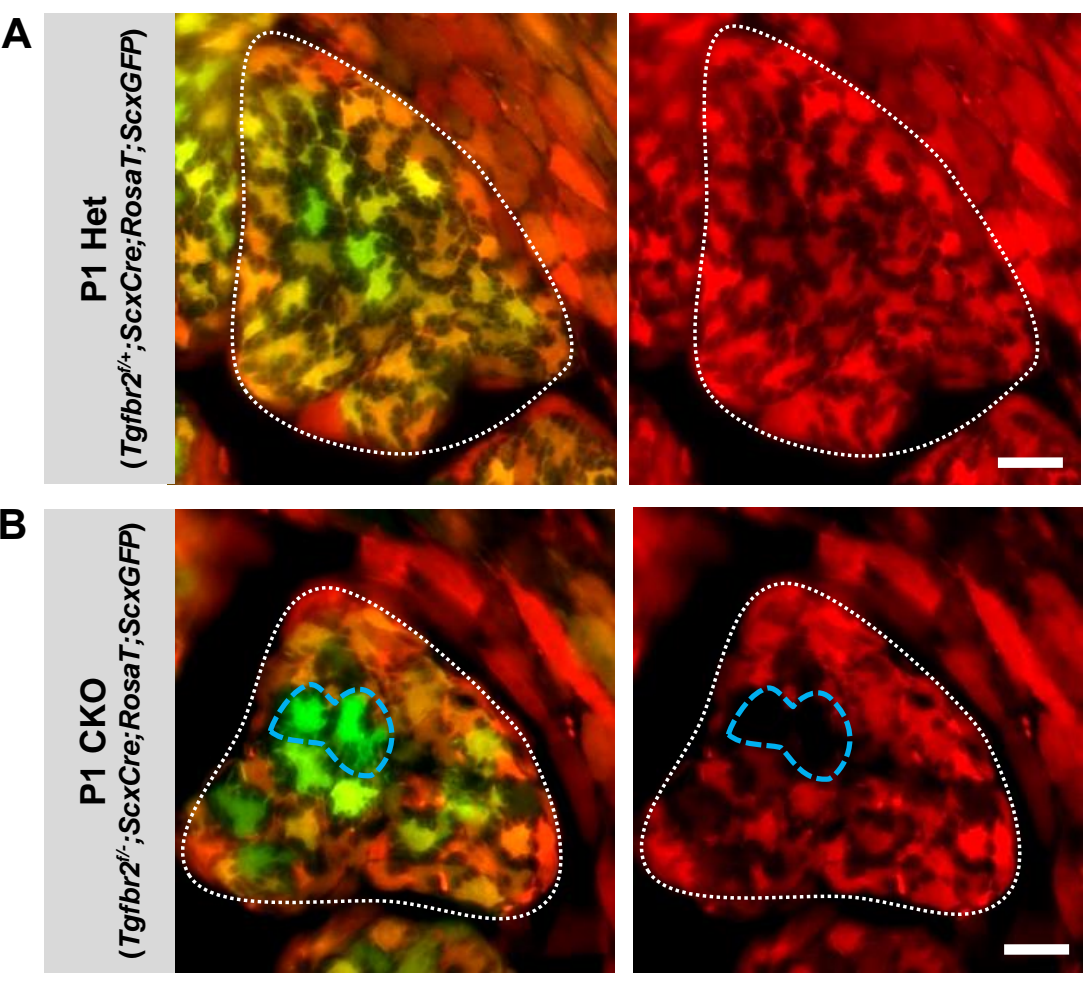

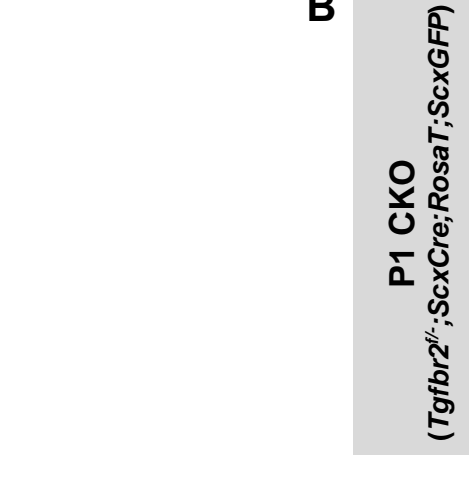

1
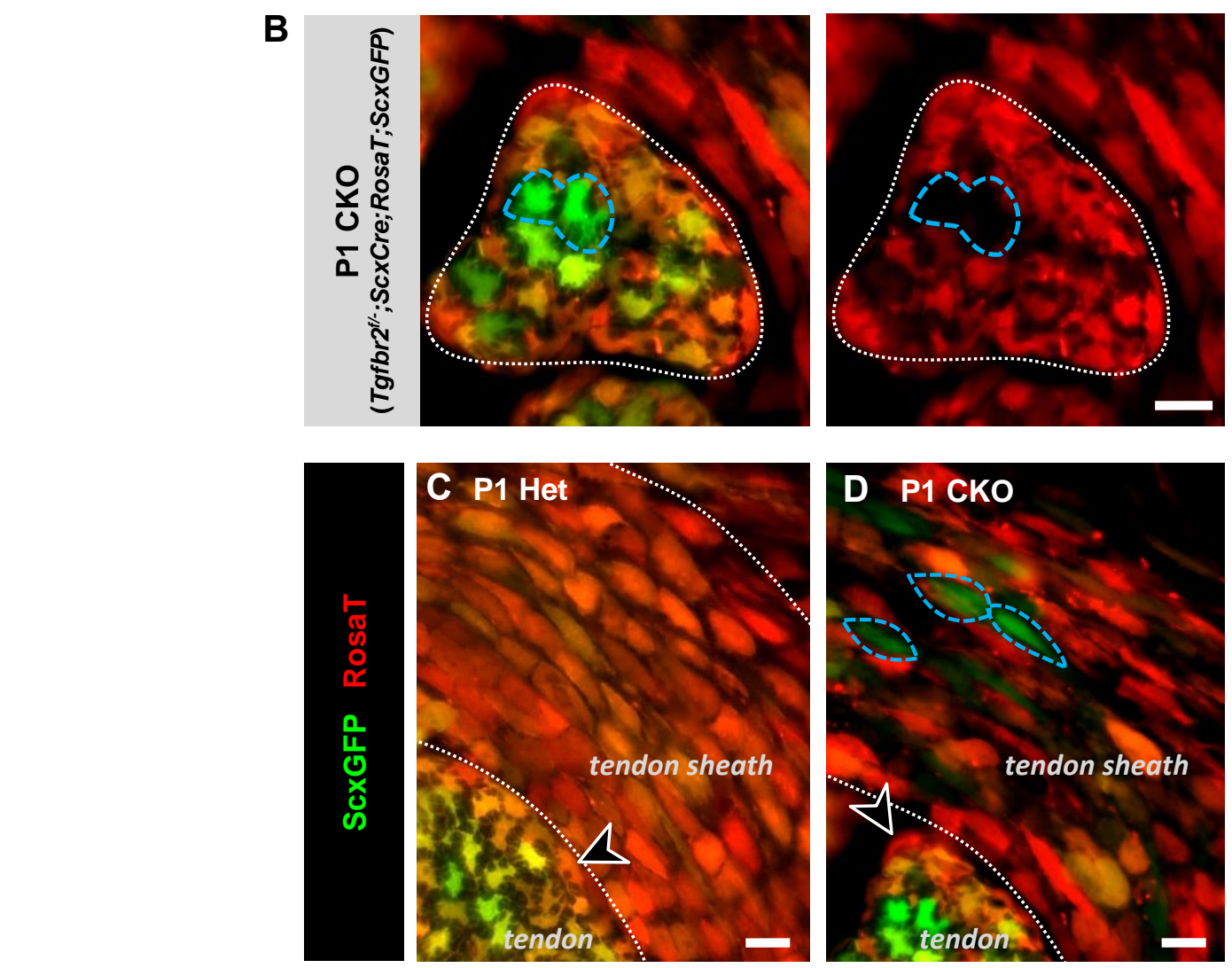

Figure 4. The recruited cells in $T g f b r 2^{S c x}$ Cre mutant tendons do not originate from peritenon or the tendon sheath. (A-D) Transverse sections of extensor digitorium communis tendons from 890 ScxGFP and RosaT-carrying mutant and heterozygous control pups at P1. $(A, B)$ In tendons of P1 891 heterozygous mice nearly all cells were positive for both ScxGFP and RosaT while ScxGFP+;RosaT892 cells were very rare (A). Conversely, there was a noticeable presence of ScxGFP+;RosaT-cells in 893 mutant tendons starting at P1, suggesting these were recruited cells in the mutant tendons. (C) 894 In Tgfbr2 $2^{f /+} ; S c x C r e$ heterozygous pups, ScxCre drives recombination of the RosaT reporter in all 895 the cells of the epitenon (black arrowhead) and tendon sheath, indicating that cells from these 
896 regions are from Scx-expressing cell lineage. The absence of RosaT expression in cell newly-

897 recruited into the mutant tendons indicates that they are not derived from these regions. (D) In

$898 T$ Tgfbr2 ${ }^{\text {ScxCre }}$ mutant pups, the newly recruited ScxGFP+;RosaT-cells could also be detected in the

899 epitenon and tendon sheath of mutant pups (blue dashed circles). Scale bar, $10 \mu \mathrm{m}$. Mutant: CKO,

900 Heterozygous: Het.

901

902

903

904

905

906

907

908

909

910

911

912

913

914

915

916

917

918

919

920

921 
P7 CKO

924

925

926

927

928

929

930

931

932

933

934

935

936

937

938

939

940

941

942

943

944

945

946

947
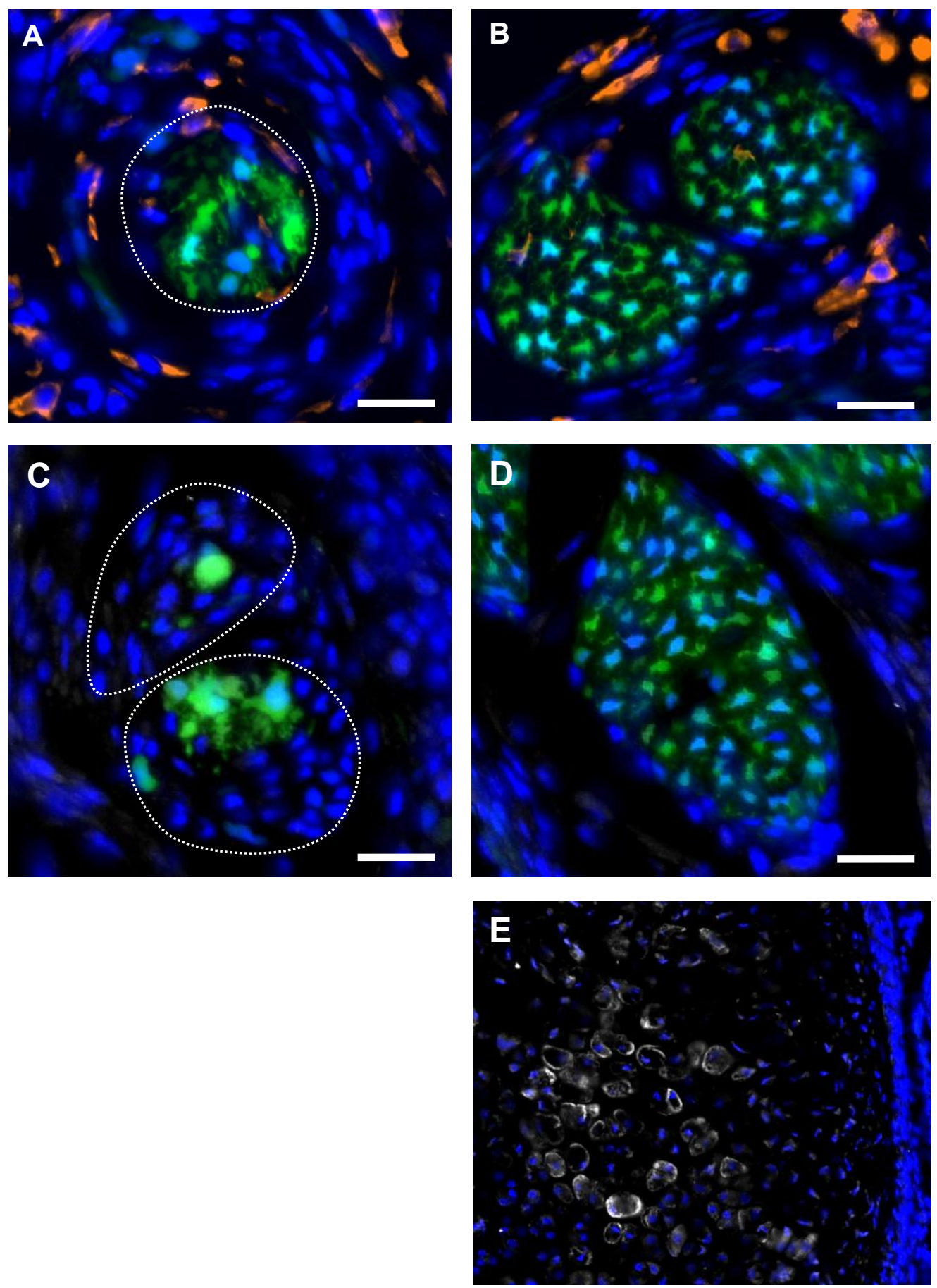

Figure 5. The inflammatory response is not activated in neonatal $T g f b r 2^{\text {ScxCre }}$ mutant tendons.

(A-E) Transverse sections of P7 forelimb tendons from mutant and littermate Het controls. In all panels DAPI nuclear staining was used to highlight cell distribution and ScxGFP labels tenocytes. $(A, B)$ Immunofluorescence staining for the macrophage-specific antigen F4/80. Only a small 
948 number of cells expressed the F4/80 antigen in both the mutant and Het control tendons. (C-E)

949 Immunofluorescence staining for the inflammatory marker TNF- $\alpha$. (C,D) Expression of TNF- $\alpha$ was

950 not detected in tendons of both the mutant and heterozygous littermate control. (E) Bone

951 marrow region on the same section as in (D) that serves as a positive control for TNF- $\alpha$ staining

952 (image not to scale). Mutant tendons are demarcated by dashed lines. Scale bar, $20 \mu \mathrm{m}$. Mutant:

953 CKO, Heterozygous: Het.

954

955

956

957

958

959

960

961

962

963

964

965

966

967

968

969

970

971

972

973

974 
975

976

977

978

979

980

981

982

983

984

985

986

987

988

989

990

991

992

993

994

995

996

997

998

999

1000

1001

1002

1003
A

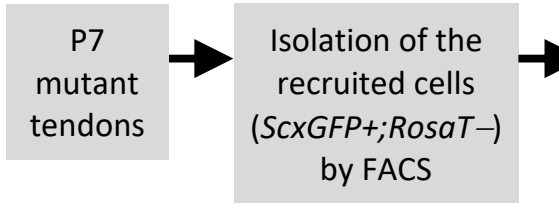

B

Percentage of Colony-

Forming Units

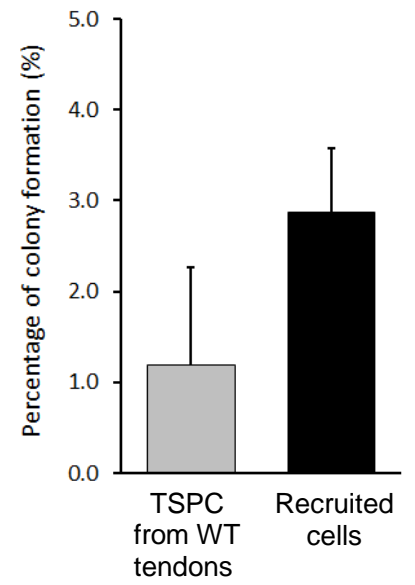

\section{TSPC from WT tendons}
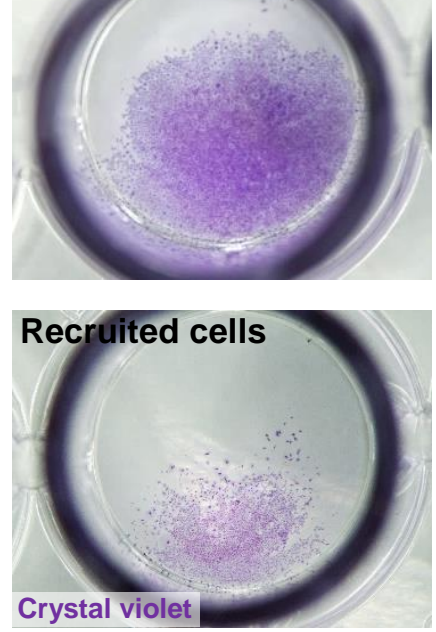

Crystal violet staining to identify formed colonies

Figure 6. The recruited cells exhibit clonogenic capability in culture. (A) Experimental outline for testing the colony forming capacity of the recruited cells. Briefly, cells were enzymaticallyreleased from the tendons of P7 mutant pups and the ScxGFP+;RosaT-newly recruited cells were isolated by FACS sorting. The cells were then seeded at one cell per well in 96-well plates and cultured for 9-14 days. Colony formation was visualized with crystal violet staining and the percentage of wells with colonies served as an indication for colony forming capacity of these cells (Tan et al., 2020). (B) About $2.9 \pm 0.7 \%$ of the ScxGFP+;RosaT-recruited cells formed colonies in cultures. For a positive control (TSPC), cells were also harvested from wild-type tendons and evaluated for their clonogenic capability under the same culture conditions. The results shown are mean \pm SD ( $n=4$ independent experiments in duplicate; a total of 768 cells were analyzed). (C) The size of colonies formed by the recruited cells isolated from Tgfbr2 $^{\text {ScxCre }}$ mutants varied and in general was smaller than those of TSPC, presumably reflecting the dynamic change in the stemness state of the recruited cells. Mutant: CKO, Wild-type: WT, Tendon-derived stem/progenitor cells: TSPC. 
1004

1005

1006

1007

1008

1009

1010

1011

1012

1013

1014

1015

1016

1017

1018

1019

1020

1021

1022

1023

1024

1025

1026

1027

1028

1029

1030

1031
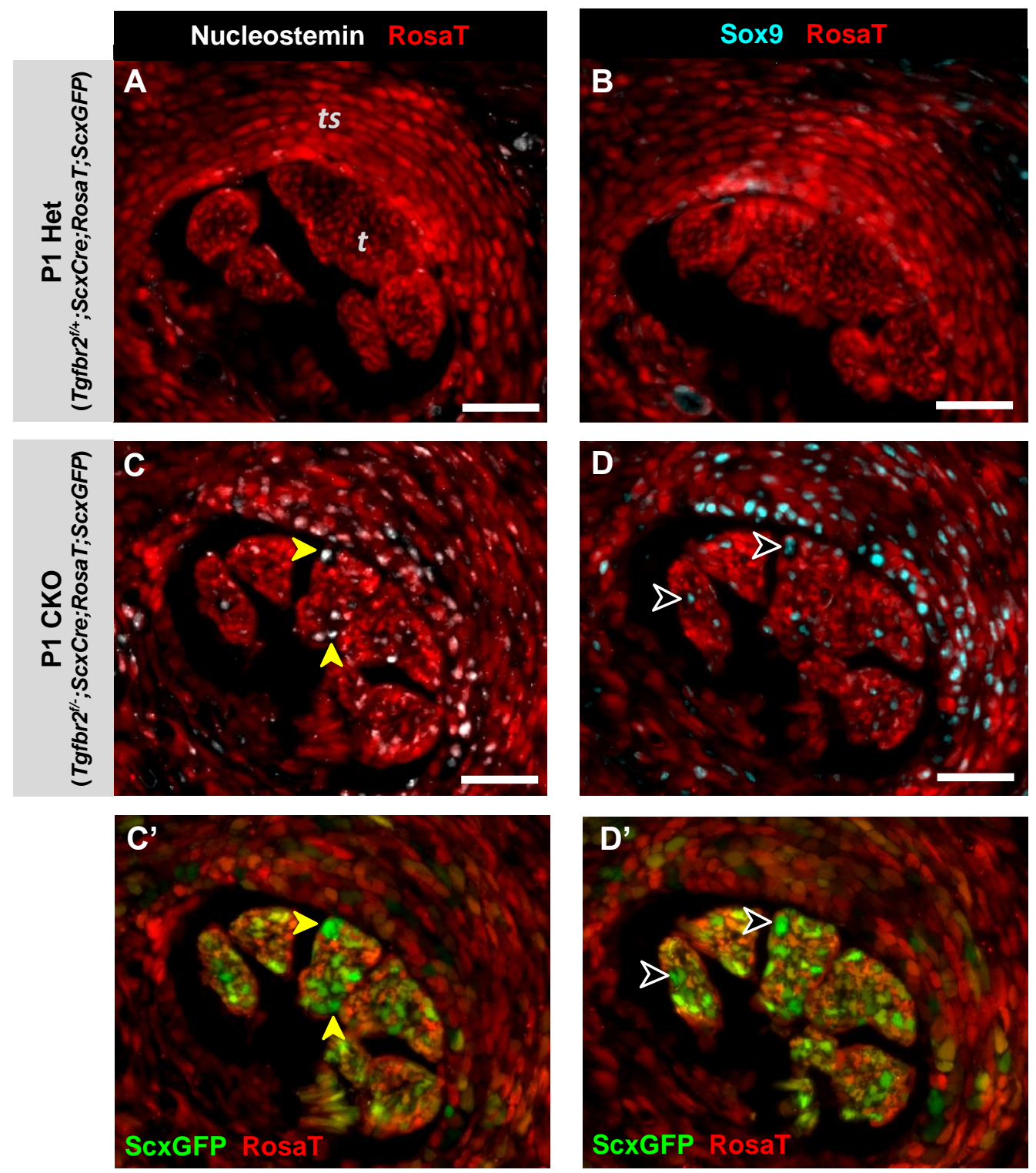

Figure 7. The recruited cells express stem/progenitor markers. Immunofluorescence staining for stem/progenitor markers on transverse sections of extensor digitorium communis tendons from forelimbs of $T g f b r 2^{S c x C r e}$ mutants and heterozygous littermates at P1, a stage with extensive cell recruitment. The ScxCre lineage was labeled with the Cre reporter RosaT. In heterozygous controls, expression of (A) nucleostemin and (B) Sox9 was either undetectable or negligible in tenocytes and the tendon sheath. (C,D) Conversely, numerous cells expressing both nucleostemin (yellow arrowheads) and Sox9 (black arrowheads) were detected within these regions in Tgfbr2 ${ }^{S c x C r e}$ mutants. $\left(C^{\prime}, D^{\prime}\right)$ represent images from the same field of view in $(C, D)$ respectively, and ScxGFP signal was captured to identify the ScxGFP+;RosaT-newly recruited cells. Scale bars, $50 \mu \mathrm{m}$. Mutant: CKO, Heterozygous: Het, Tendon sheath, ts, Tendons, t. 


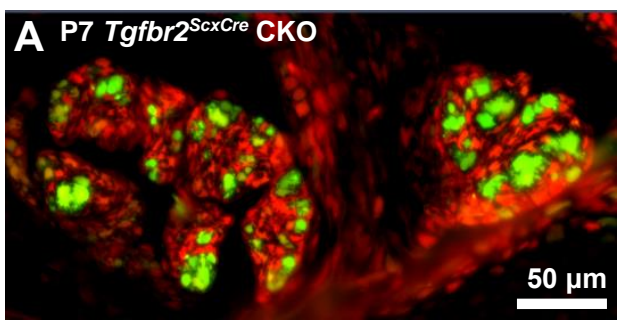

\section{Mean percentage of recruited} cells in mutant tendons

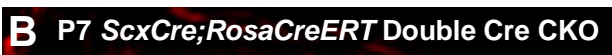

1040
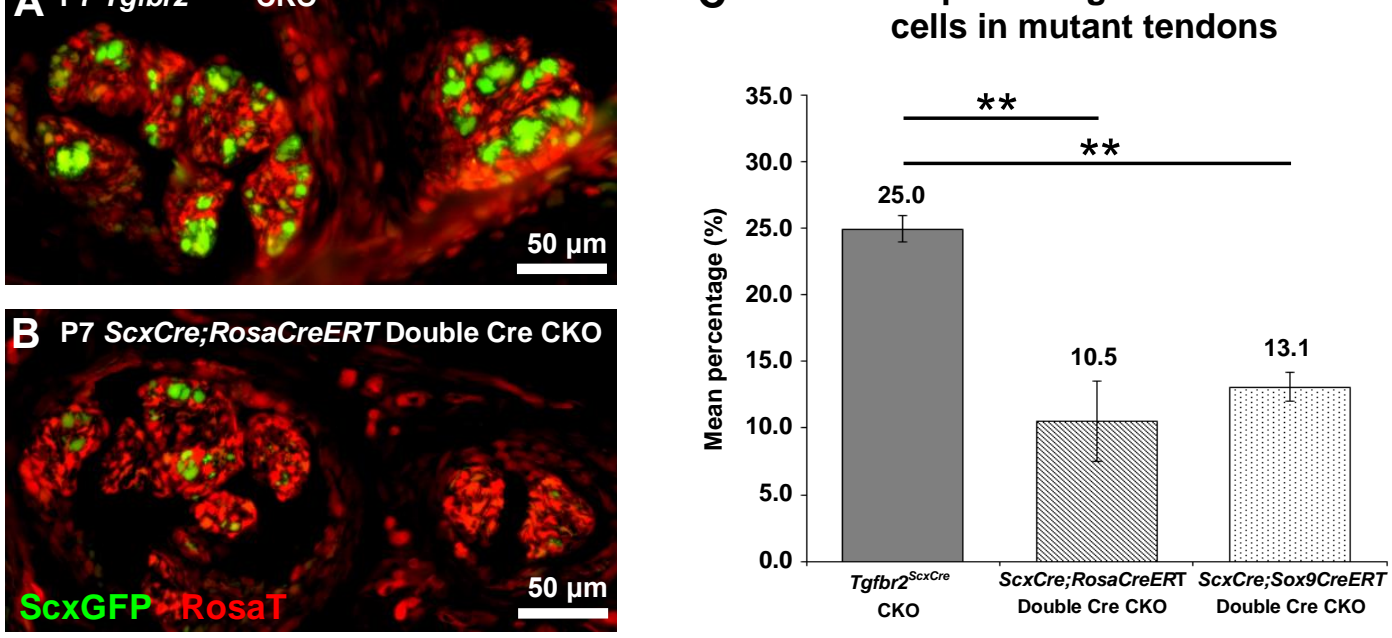

\section{P7 ScxCre;RosaCreERT Double Cre CKO (Tgfbr2f/;ScxCre;RosaCreERT2)}

1049
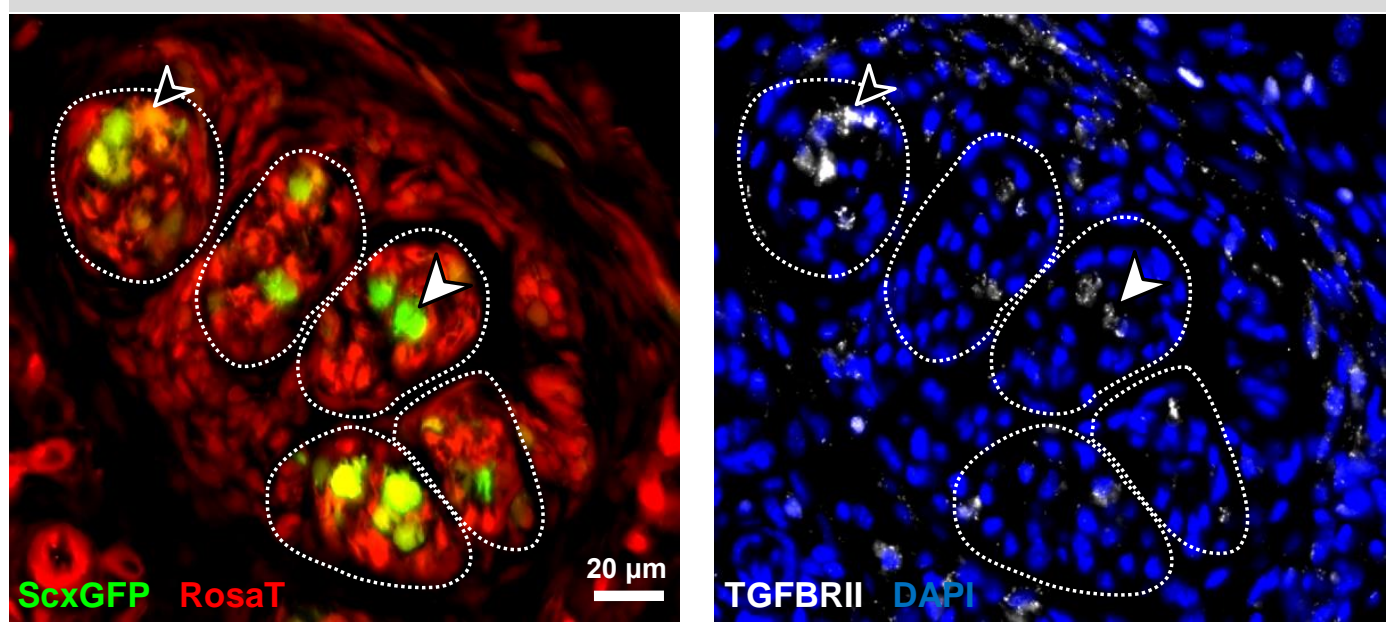

1050

Figure 8. Cell autonomous TGF $\beta$ signaling is essential for cell recruitment into mutant tendons. The ubiquitous RosaCreERT2 driver was incorporated into the Tgfbr $2^{\text {ScxCre }}$ mutant background (Tgfbr2f/-;ScxCre;RosaCreERT2, called hereafter ScxCre;RosaCreERT) to examine the effect of global loss of the TGF $\beta$ signaling on cell recruitment into Tgfbr2 ${ }^{S c x C r e}$ mutant tendons. Tamoxifen was administered at P1 and P2, and the effects on cell recruitment were evaluated at P7. $(A, B)$ Transverse sections through the extensor digitorium communis tendons from single Cre (Tgfbr2 ${ }^{\text {ScxCre }}$ ) and ScxCre;RosaCreERT Double Cre littermates. Qualitative histological analysis reveals a dramatic reduction in the number of recruited cells (ScxGFP-expressing cells) in the Double Cre mutant pups compared to Tgfbr $2^{S c x C r e}$ mutants. (C) Quantitative determination of the number of recruited cells in P7 experimental pups. There was a nearly 60\% reduction in cell recruitment in the ScxCre; RosaCreERT Double Cre mutant pups, suggesting that TGF $\beta$ signaling is 
1060

1061

1062

1063

1064

1065

1066

1067

1068

1069

1070

1071

1072

1073

1074

1075

1076

1077

1078

1079

1080

1081

1082

1083

1084

1085

1086

1087

essential for cell recruitment. For cell lineage study, the Double Cre strategy was also employed to target Tgfbr2 specifically in Sox9-expressing cells (Tgfbr2 ${ }^{f /-} ; \mathrm{ScxCre}$;Sox9CreERT2, called hereafter ScxCre;Sox9CreERT). The results showed about 48\% decrease in recruited cell numbers, suggesting that the recruited cells are from Sox9-expressing cell lineage. The results shown are mean \pm SD ( $n=3, * * p<0.01)$. (D) Immunofluorescence staining for TGFBRII on transverse sections of ScxCre;RosaCreERT Double Cre mutant forelimbs. The left panel shows the overlay of the green (SCXGFP) and red (RosaT) channel, and the right panel shows for the same section the expression of the TGFBRII on the background of DAPI nuclear counterstain. The ScxGFP-expressing cells that were still recruited into Double Cre mutant tendons were also positive for the receptor (arrowheads), suggesting a complete dependence of cell recruitment on TGF $\beta$ signaling. Mutant: CKO.

.

3

4

(1)

(6)



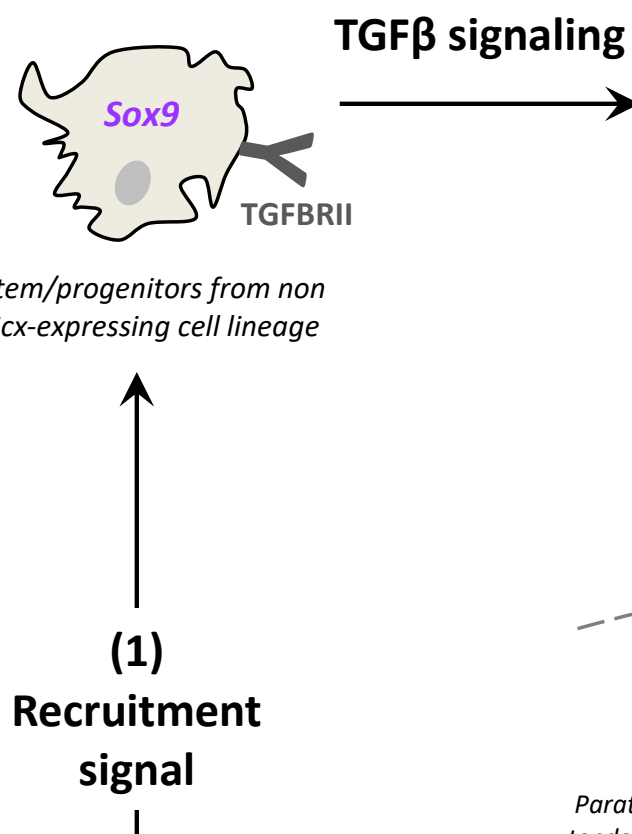

Figure 9. Proposed model for cell recruitment process into Tgfbr2 ${ }^{\text {Scxcre }}$ mutant tendons. (1) The degenerating mutant tendons emit a recruitment signal. (2) The recruitment signal leads to activation of TGF $\beta$ signaling and to recruitment of a population of Sox9-positive and Scx-negative stem/progenitor cells. The ScxCre-lineage tracing suggests that these cells are not derived from surrounding peritenon or tendon sheath. (3) The activated cells are recruited towards the mutant tendon. (4) These Sox9-expressing cells turned on tendon markers and became ScxGFP-positive only upon entering the mutant tendons. Notably, the newly recruited cells, identified as

1112 ScxGFP+;RosaT-cells in this study, could be detected as early as P1 in the mutant tendons, 1113 peaked between P1 to P3 and remained detectable throughout the study period. These cells also

1114 express nucleostemin (NS), a stem/progenitor marker that has been reported in tendon-derived 1115 stem/progenitor cells but not in mature tenocytes. TGF $\beta$ type II receptor: TGFBRII. 
A

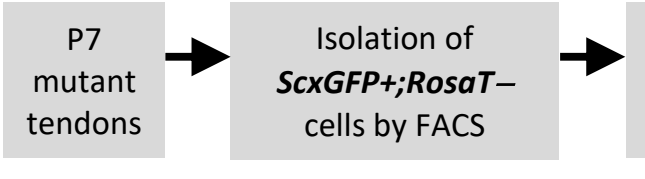

Cultured in 8 well chamber slides

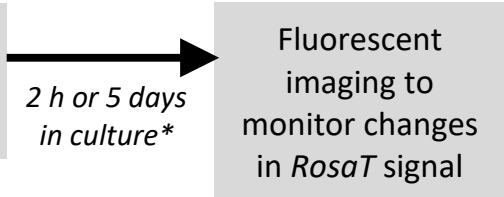

in RosaT signal delay.
B

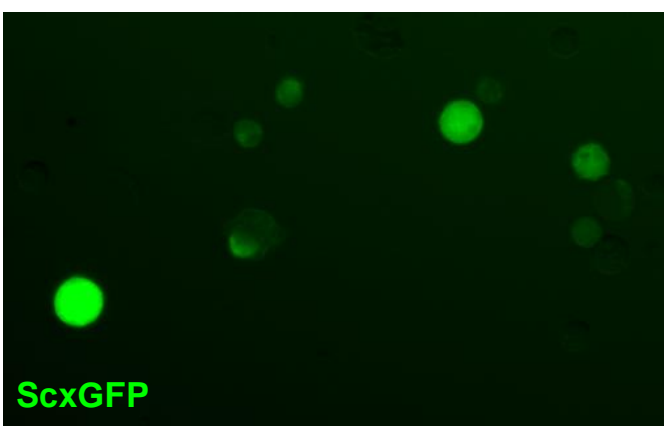

C
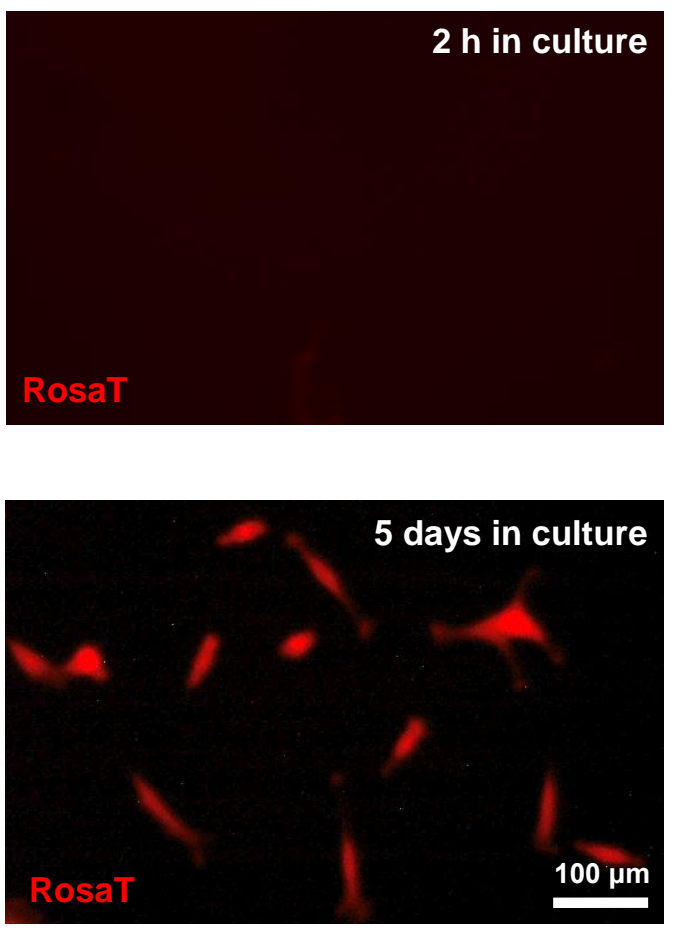

* Cells were cultured in high glucose Dulbecco's modified Eagle's medium (DMEM) containing 2mM L-glutamine, $1 \%$ penicillin/streptomycin and $20 \%$ fetal bovine serum. The cultures were incubated at $37^{\circ} \mathrm{C}$ in a humidified incubator containing $5 \% \mathrm{CO}_{2}$.

\section{Supplementary Figure 1. Newly recruited ScxGFP+;RosaT- cells subsequently induce} expression of the Cre reporter. (A) Experimental outline: Cells were enzymatically-released from P7 Tgfbr2 ${ }^{\text {ScxCre }}$ mutant tendons. ScxGFP+;RosaT-cells were then sorted by FACS and cultured in 8-well chamber slides. Fluorescence imaging was carried out at designated time-points to monitor changes in RosaT signal. (B) Fluorescent images of the sorted cells after $2 \mathrm{~h}$ in culture. Note that most cells were still floating in medium. As determined by the FACS gating, only ScxGFPpositive and RosaT-negative cells were isolated. Images not to scale. (C) Brightfield and fluorescent images of the sorted cells after 5 days of culture, in which all of the cells have now expressed RosaT Cre reporter. These findings support the hypothesis that, upon induction of the Scx enhancer, the ScxGFP signal will be detected first and the RosaT signal will follow with some 


\section{P7 CKO}
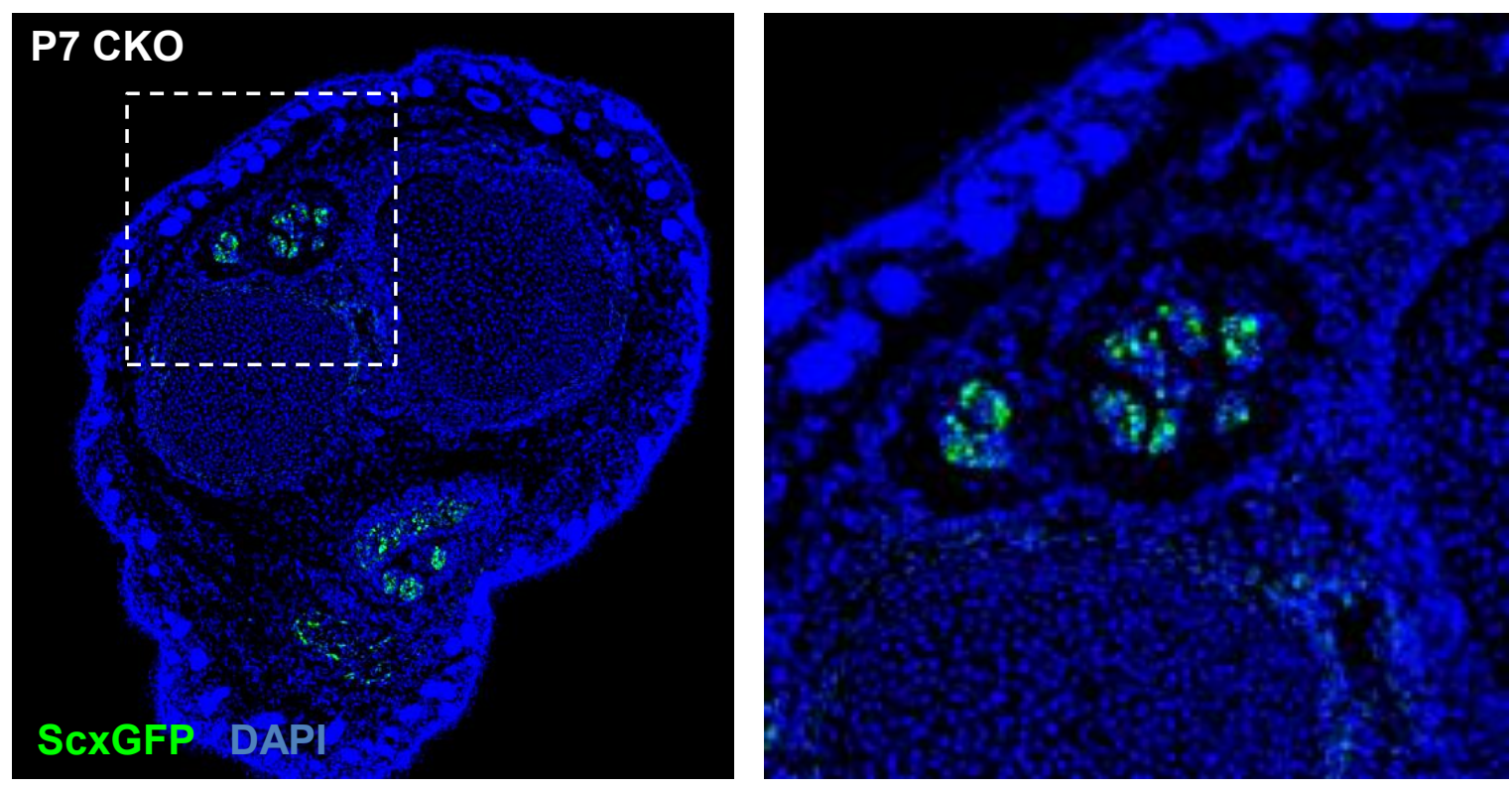

1154 Supplementary Figure 2. The recruited cells turned on tendon gene expression only upon

1155 arrival to or entering the tendon. Histological examination of transverse sections of $T g f b r 2^{S c x C r e}$

1156 mutant forelimbs stained with DAPI to visualize cell distribution in the section and ScxGFP to

1157 identify the recruited cells. Shown here is a representative histological section from P7 mutants.

1158 Recruited cells expressing the tendon reporter ScxGFP were not detected away from the tendons

1159 but only within or adjacent to mutant tendons, suggesting that irrespective of their origin the

1160 cells turned on tendon gene expression only upon arrival to or entering the tissue. Boxed region

1161 is shown enlarged in the right panel. Images are not to scale. 